\title{
COMPLETION OF REAL FANS AND ZARISKI-RIEMANN SPACES
}

\author{
GÜNTER EWALD AND MASA-NORI ISHIDA*
}

(Received April 14, 2004, revised Novemer 1, 2004)

\begin{abstract}
Given a real fan in a real space consisting of real convex polyhedral cones, we construct a complete real fan which contains the fan, by two completely different methods. The first one is purely combinatorial and a proof of a related version was sketched earlier by Ewald. The second one is based on Nagata's method of imbedding an abstract variety into a complete variety. For the second method, we introduce the theory of Zariski-Riemann space of a fan.
\end{abstract}

Introduction. A fan in a real space is defined as a cell complex consisting of polyhedral cones with the apex at the origin. A finite fan $\Sigma$ is said to be complete if the union of cones in $\Sigma$ is the whole space. The theory of toric varieties says that, to each finite fan consisting of rational polyhedral cones, is associated a toric variety, and the fan is complete if and only if the toric variety is complete (see, for example, Ewald [E], Fulton [F2], Oda [O1]). Nagata's compactification theorem says that any algebraic variety can be embedded in a complete algebraic variety [N1, Theorem 4.3]. This theorem was generalized for normal algebraic varieties with algebraic group actions by Sumihiro [S1], i.e., the equivariant completion theorem. By using Sumihiro's theorem, we can complete a rational fan $\Sigma$ as follows Let $X$ be the toric variety associated to $\Sigma$. Since $X$ is a normal variety with torus action, there exists an equivariant completion $\bar{X}$. Since $\bar{X}$ is a complete toric variety, it corresponds to a complete fan $\bar{\Sigma}$. Then $\bar{\Sigma}$ is a completion of $\Sigma$.

Since it is a quite simple problem on convex polyhedral sets in a real space, we would like to avoid this roundabout proof. In this paper, we give two different direct proofs which are valid for not necessarily rational fans. The first proof given in Section 1 is purely combinatorial and was sketched in [E, Theorem 2.8] in the case of a rational fan.

The second one is done by using Nagata's method applied for fans. In Nagata's proof, the Zariski-Riemann space, i.e., the topological space of all valuation rings of the function field plays an important role. The Zariski-Riemann space was introduced originally by Zariski [Z1], [Z2] for the theory of local uniformization of algebraic varieties. In Section 2, we define the Zariski-Riemann spaces for rational fans. We discuss on blowups of not necessarily rational fans in Section 3. Using the results of these sections, the existence of the completion is first proved for rational fans in Section 4. In the last section, the definition of the ZariskiRiemann spaces is generalized for $\boldsymbol{k}$-fans for any subfield $\boldsymbol{k}$ of $\boldsymbol{R}$. Theorem 5.4 claims that any

2000 Mathematics Subject Classification. Primary 14M25; Secondary 52B20.

*Partly supported by the Grant-in-Aid for Scientific Research (C), Japan Society for the Promotion of Science. 
finite $\boldsymbol{k}$-fan is embedded in a complete $\boldsymbol{k}$-fan. The theorem applied for $\boldsymbol{k}=\boldsymbol{R}$ is the second proof of the completion theorem for real fans.

\section{Combinatorial proof.}

Notation. Given a set $E$ of vectors in $\boldsymbol{R}^{n}$ we denote by $\operatorname{pos} E$ the set of all linear combinations of elements of $E$ with non-negative coefficients, and call it the positive hull of $E$. If $E$ is finite, we say pos $E$ is a (real) polyhedral cone $\sigma$. The dimension $\operatorname{dim} \sigma$ of $\sigma$ is defined to be the dimension of the linear hull $\operatorname{lin} \sigma$ of $\sigma$. If $\sigma$ has linearly independent generators, we call it a simplicial cone. If $\sigma$ has rational generators, we call $\sigma$ rational. By a face of $\sigma$ we mean an intersection $\sigma \cap H$, where $H$ is a (linear) hyperplane such that $\sigma$ is totally contained in one of the two closed half-spaces bounded by $H$. If $\{0\}$ is a face of $\sigma$, we call it the apex of $\sigma$.

A collection $\Sigma$ of (real) polyhedral cones with apex $\{0\}$ is said to be a (real) fan if it is a cell complex, that is, (i) each face of a cone of $\Sigma$ is also in $\Sigma$, (ii) the intersection of two cones of $\Sigma$ is a common face of the cones. We call $\Sigma$ rational if all cones are rational. If $\Sigma$ and $\Sigma^{\prime}$ are fans in $\boldsymbol{R}^{n}$ and $\Sigma \subset \Sigma^{\prime}$, we say $\Sigma$ is a subfan of $\Sigma^{\prime}$. By the $\operatorname{star} \operatorname{st}(\sigma, \Sigma)$ of a cone $\sigma \in \Sigma$ in $\Sigma$ we mean the set of all cones $\tau \in \Sigma$ such that $\sigma \subset \tau$. The support $|\Sigma|$ of $\Sigma$ is the union of the cones in $\Sigma$. If $\Sigma$ is finite and $|\Sigma|$ equals $\boldsymbol{R}^{n}$, we say $\Sigma$ is complete, and a completion of any of its subfans. If a finite fan $\Sigma$ is not complete, we call the collection of cones of $\Sigma$ which lie in the (topological) boundary of $|\Sigma|$ the boundary bd $\Sigma$ of $\Sigma$. Clearly, bd $\Sigma$ is again a fan. Given $\varepsilon>0$, the $\varepsilon$-neighborhood of $\Sigma$ is defined as the union of all 1 -cones $\operatorname{pos}\{a\}$ where $a$ is a unit vector representing a point of distance less than $\varepsilon$ from $|\Sigma|$.

As a specific type of cones we consider the following. If $\rho$ is a 1-dimensional cone not contained in the linear hull of a cone $\sigma$, we call $\sigma . \rho=\operatorname{pos}(\sigma \cup \rho)$ a pyramid with apex $\rho$ over the basis $\sigma$. Clearly, $\operatorname{dim}(\sigma . \rho)=1+\operatorname{dim} \sigma$. A pyramid over a pyramid is said to be a twofold pyramid or a 2-pyramid, and a pyramid over a $(k-1)$-pyramid inductively a $k$-fold pyramid or a $k$-pyramid. $\sigma$ is considered a 0 -fold pyramid over itself. $k$-fold pyramids can be written as

$$
\sigma \cdot \rho_{1} \cdot \cdots \cdot \rho_{k}=\sigma \cdot \tau,
$$

where $\tau=\rho_{1}, \cdots \rho_{k}$ is a simplicial cone. If $P$ is a polytope or a polyhedral set and $p^{1}, \ldots, p^{k}$ are vectors (representing points) such that $\sigma=\operatorname{pos} P$ and $\rho_{i}=\operatorname{pos} p^{i}, i=$ $1, \ldots, k$, then $\sigma \cdot \tau=\operatorname{pos}\left(P \cdot p^{1} . \cdots \cdot p^{k}\right)$, where $P \cdot p^{1} . \cdots \cdot p^{k}$ is an ordinary $k$-fold pyramid (unbounded if $P$ is not a polytope).

1.1. Main result. All fans in this section are assumed to be finite.

THEOREM 1.1. Every real fan $\Sigma$ can be completed.

In [E, Theorem 2.8], we have sketched the proof of this theorem for rational fans. In this section, we present an explicit proof in the general case. The second proof of this theorem is given in Section 5 (cf. Theorem 5.4).

PROOF OF THEOREM 1.1. For the purpose of our proof it is useful to show a somewhat stronger version of Theorem 1.1: 
THEOREM 1.2. Given a fan $\Sigma$ and an $\varepsilon>0$, there exists a fan $\Sigma_{0}$ and a complete fan $\Sigma^{\prime}$ such that the following are satisfied:

(1) $\Sigma \subset \Sigma_{0} \subset \Sigma^{\prime}$.

(2) $\Sigma_{0} \backslash \Sigma$ consists of the multifold pyramids joining the cones of $\Sigma$ to simplicial cones in bd $\Sigma_{0}$, and of the faces of such pyramids.

(3) If $\sigma \in \Sigma^{\prime}$ intersects the cells of $\Sigma$ only in 0 , then $\sigma$ is a simplicial cone.

(4) $|\Sigma| \backslash\{0\}$ lies in the interior of $\left|\Sigma_{0}\right|$.

(5) $\left|\Sigma_{0}\right|$ is contained in the $\varepsilon$-neighborhood of $\Sigma$.

We apply induction on $n$. For $n=1$, either $\Sigma=\{\{0\}\}$ or $\Sigma=\{\{0\}, \operatorname{pos}\{1\}\}$ or $\Sigma=$ $\{\{0\}, \operatorname{pos}\{-1\}\}$ or $\Sigma=\{\{0\}, \operatorname{pos}\{1\}, \operatorname{pos}\{-1\}\}$, the last fan being the completion of all the others. If $n=2$, let $S$ be the unit circle. We may assume $\{0\}$ not to be the only cone of $\Sigma$, any complete fan being a completion of $\Sigma$ in this case. So $\Sigma$ splits $S$ into finitely many (closed) circular arcs. Let $C$ be one of these arcs and $p, q$ its end points. We choose points $p^{\prime}, q^{\prime}$ in the relative interior of $C$ so that the distances between $p, p^{\prime}$ and $q, q^{\prime}$, respectively, are less than $\varepsilon / 2$, and that the cones $\operatorname{pos}\left\{p, p^{\prime}\right\}, \operatorname{pos}\left\{q, q^{\prime}\right\}$ intersect only in $\{0\}$. We extend $\Sigma$ by adding $\operatorname{pos}\left\{p, p^{\prime}\right\}, \operatorname{pos}\left\{p^{\prime}\right\}, \operatorname{pos}\left\{q, q^{\prime}\right\}, \operatorname{pos}\left\{q^{\prime}\right\}$. Doing this for all arcs (and assuming $\varepsilon$ small enough to begin with), we obtain a fan $\Sigma_{0}$. Now $S \backslash\left|\Sigma_{0}\right|$ consists of finitely many arcs. If one of the arcs has length $\pi$ or more, we split it into two arcs of length less than $\pi$. We add to $\Sigma$ the closed angular regions determined by the arcs and their boundary 1-cones and obtain a complete fan $\Sigma^{\prime}$. It is readily verified that $\Sigma, \Sigma_{0}$ and $\Sigma^{\prime}$ satisfy (1) through (5).

Let $n>2$. Again we may assume that $\{0\}$ is not the only cone of $\Sigma$. So let $\rho$ be a 1-cone of $\Sigma$ for which $\operatorname{st}(\rho, \Sigma)$ is not complete (if none of such exists, $\Sigma$ is already complete). Let $H$ be the (affine) tangent hyperplane of the unit sphere $S$ at $a=\rho \cap S$. Then $H$ intersects the cones of $\Sigma$ either not at all or in convex polytopes (if bounded) or so-called polyhedral sets (if unbounded). In Figure 1 we illustrate the case $n=3$ (heavy lines and hatched regions). Let $d$ be the smallest distance that $a$ has from the cones of $\Sigma \backslash \operatorname{st}(\rho, \Sigma)$. Clearly $d>0$. We consider the $(n-2)$-sphere $S_{a}$ of radius at most $d / 2$ and center $a$ in $H$. For the moment we regard $a$ as the origin of the $(n-1)$-space $H$. By $\operatorname{pos}_{a}$ let us denote the positive hull with respect to this origin.

$$
\Sigma_{a}:=\left\{\operatorname{pos}_{a}(H \cap \sigma) ; \sigma \in \operatorname{st}(\rho, \Sigma)\right\}
$$

is then a fan in $H$. It represents the quotient fan $\Sigma / \rho$ (up to a translation; compare Ewald [E, p. 81, Definition 3.3]).

Now we apply the inductive assumption to $\Sigma_{a}$ and obtain for any $\varepsilon_{a}>0$ fans $\Sigma_{a, 0}, \Sigma_{a}^{\prime}$ satisfying (1)-(5) (with terms indexed by $a$, and $d / 2$ considered as unit length). We wish to construct an extension of $\Sigma$ by using $\Sigma_{a, 0}, \Sigma_{a}^{\prime}$. Since the latter fans collide, in general, with cones of $\Sigma \backslash \operatorname{st}(\rho, \Sigma)$, we first construct a map which assigns to each cone of $\Sigma_{a}^{\prime}$ a polytope or polyhedral set contained in the cone:

I. Let $\sigma_{a} \in \Sigma_{a}$, hence $\sigma_{a}=\operatorname{pos}_{a}(H \cap \sigma)$ for some $\sigma \in \operatorname{st}(\rho, \Sigma)$. Then we assign

$$
\sigma_{a} \mapsto \phi_{a}\left(\sigma_{a}\right)=H \cap \sigma
$$




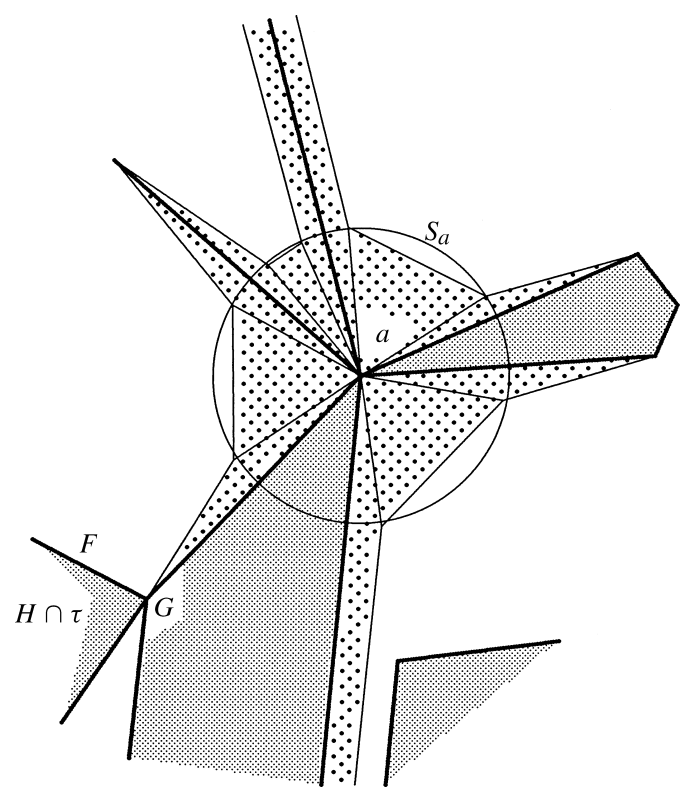

FIGURE 1.

II. Let $\sigma_{a} \in \Sigma_{a, 0} \backslash \Sigma_{a}$ be a multifold pyramid $\sigma_{a, o} . \rho_{a, 1} \cdots \rho_{a, k}$, where $\sigma_{a, o} \in \Sigma_{a}$, $\sigma_{a, o}=\operatorname{pos}_{a}\left(H \cap \sigma_{o}\right)$, and $\rho_{a, i}=\operatorname{pos}_{a}\left\{p_{a, i}\right\}, p_{a, i} \in S_{a}, i=1, \ldots, k$. We assign

$$
\sigma_{a} \mapsto \phi_{a}\left(\sigma_{a}\right)=\operatorname{clconv}\left(\left(H \cap \sigma_{o}\right) \cup\left\{p_{a, 1}\right\} \cup \cdots \cup\left\{p_{a, k}\right\}\right),
$$

where "cl" means the "topological closure" (needed if $H \cap \sigma_{o}$ is unbounded).

III. Let $\sigma_{a} \in \Sigma_{a, 0} \backslash \Sigma_{a}$ and $\sigma_{a} \cap\left|\Sigma_{a}\right|=\{a\}$ or $\sigma_{a} \in \Sigma_{a}^{\prime} \backslash \Sigma_{a, 0}$. Then $\sigma_{a}=$ $\operatorname{pos}_{a}\left\{p^{1}, \ldots, p^{r}\right\}$, where $p^{1}, \ldots, p^{r}$ are in $S_{a}$ and linearly independent (with respect to $H$ as a linear space). We set

$$
\sigma_{a} \mapsto \phi_{a}\left(\sigma_{a}\right)=\operatorname{conv}\left\{a, p^{1}, \ldots, p^{r}\right\} \quad(\text { an }(r+1) \text {-simplex }) .
$$

In Figure 1 the dotted regions illustrate the $\phi_{a}\left(\sigma_{a}\right)$ of type II or III.

IV. $\phi_{a}(\{a\})=\{a\}$.

LEMMA 1.3. $\phi_{a}$ as defined by I-IV maps $\Sigma_{a}^{\prime}$ onto a cell complex A consisting of polytopes and polyhedral sets in $H$. It has the following properties:

(a) $\phi_{a}$ is bijective and preserves inclusions.

(b) $\operatorname{pos}_{a} \phi_{a}\left(\sigma_{a}\right)=\sigma_{a}$ for all $\sigma_{a} \in \Sigma_{a}^{\prime}$.

(c) $\varepsilon_{a}>0$ can be chosen so that for any $\tau \in \Sigma \backslash \operatorname{st}(\rho, \Sigma)$ we have: $\tau \cap|A|$ is empty or contained in $\phi_{a}\left(\sigma_{a}\right)$ for some $\sigma_{a} \in \Sigma_{a}$.

PRoof. (a) and (b) readily follow from the definitions I-IV. In order to show (c) we recall an elementary fact from convex polytope theory: (*) If $F, G$ are (closed) polytopes or 
polyhedral sets in $\boldsymbol{R}^{n}$ such that $F \cap G$ is empty, then $F$ and $G$ have positive distance, that is, there exists a $d>0$ such that each point of $F$ has at least distance $d$ from $G$. Suppose (c) is false. Then there exists a $\tau \in \Sigma \backslash \operatorname{st}(\rho, \Sigma)$ and an $x$ in $\tau \cap|A|$ but not in $\phi_{a}\left(\sigma_{a}\right)$ for any $\sigma_{a} \in \Sigma_{a}$. Since the $\phi_{a}\left(\sigma_{a}\right)$ of type III are contained in the ball $B_{a}, x$ cannot lie in one of such. So $x$ lies in a $k$-fold pyramid $\phi_{a}\left(\sigma_{a, 0} \cdot p^{1} . \cdots \cdot p^{k}\right)$ over $\phi_{a}\left(\sigma_{a, 0}\right)$, where $\sigma_{a, 0} \in \Sigma_{a}$ but $x$ does not lie in the basis $\phi_{a}\left(\sigma_{a, 0}\right)$ of the pyramid.

Let $F$ be the smallest face of $\tau \cap H$ which contains $x$ (in its relative interior). If $F \cap$ $\phi_{a}\left(\sigma_{a, 0}\right)=\emptyset$, then $F$ has, by $(*)$, positive distance from $\phi_{a}\left(\sigma_{a, 0}\right)$, and we choose $\varepsilon_{a}$ to be at most half this distance. Then $x$ cannot lie in the above pyramid. So let $F \cap \phi_{a}\left(\sigma_{a, 0}\right)$ be nonempty. Since $F$ and $\phi_{a}\left(\sigma_{a, 0}\right)$ are intersections of cones of $\Sigma$ and $H, F \cap \phi_{a}\left(\sigma_{a, 0}\right)$ is a common face $G$ of $F$ and $\phi_{a}\left(\sigma_{a, 0}\right)$. Let $\operatorname{dim} G=m$. Then $G, a$, and $x$ span an $(m+2)$ dimensional affine space in which the hyperplanes spanned by $G, a$ and $G, x$, respectively, have an angle $\alpha>0$ (see the illustration in Figure 1). Since $x$ lies in the $k$-fold pyramid $\phi_{a}\left(\sigma_{a, 0}\right) \cdot p^{1} \ldots . p^{k}$ over $\phi_{a}\left(\sigma_{a, 0}\right)$, the angle $\alpha$ could be made arbitrarily small by choosing $\varepsilon_{a}$ small enough, a contradiction to $\alpha>0$ being given. So let $\varepsilon_{a}$ be chosen appropriately. Since our arguments apply to finitely many faces, we may select the smallest $\varepsilon_{a}$ among those which occur as a common bound. This proves Lemma 1.3.

Now we define the following map $\psi_{a}$ on $A$ :

I'. If $\sigma \in \Sigma$ and $H \cap \sigma \in A$, we assign

$$
H \cap \sigma \mapsto \psi_{a}(H \cap \sigma)=\sigma .
$$

II'. If $\phi_{a}\left(\sigma_{a}\right)=\operatorname{clconv}\left(\left(H \cap \sigma_{o}\right) \cup\left\{p_{a, 1}\right\} \cup \cdots \cup\left\{p_{a, k}\right\}\right)$ according to II, we consider the point $p_{a, i}$ as vector $q_{i}=a+p_{a, i}$ in $\boldsymbol{R}^{n}$ and assign for $\rho_{i}=\operatorname{pos}\left\{q_{i}\right\}, i=1, \ldots, k$

$$
\phi_{a}\left(\sigma_{a}\right) \mapsto \psi_{a}\left(\phi_{a}\left(\sigma_{a}\right)\right)=\sigma_{o} . \rho_{1} \cdot \cdots \cdot \rho_{k} .
$$

III'. If $\phi_{a}\left(\sigma_{a}\right)=\operatorname{conv}\left\{a, p^{1}, \ldots, p^{r}\right\}$ according to III, we consider again the points $p^{i}$ as vectors $q^{i}=a+p^{i}$ in $\boldsymbol{R}^{n}$ and assign for $\rho^{i}=\operatorname{pos}\left\{q^{i}\right\}, i=1, \ldots, r($ and $\rho=\operatorname{pos} a$ )

$$
\phi_{a}\left(\sigma_{a}\right) \mapsto \psi_{a}\left(\phi_{a}\left(\sigma_{a}\right)\right)=\rho . \rho^{1} . \cdots . \rho^{r} .
$$

$\mathrm{IV}^{\prime}$.

$$
\{a\} \mapsto \psi(\{a\})=\rho .
$$

LEMMA 1.4. If we add to $\Sigma$ all cones $\psi_{a}\left(\phi_{a}\left(\sigma_{a}\right)\right)$ and their faces for $\sigma_{a} \in \Sigma_{a}^{\prime} \backslash \Sigma_{a}$, then we obtain a set $\Sigma^{(1)}$ which is a fan, provided $\varepsilon_{a}$ is chosen small enough.

PROOF. By construction, $\psi_{a}$ clearly is bijective. We must show that for sufficiently small $\varepsilon_{a}$ two cones $\tau, \tau^{\prime}$ of $\Sigma^{(1)}$ intersect in a common face of $\tau, \tau^{\prime}$ which belongs to $\Sigma^{(1)}$. Since $\Sigma \subset \Sigma^{(1)}$, this is true if $\tau, \tau^{\prime}$ both lie in $\Sigma$. Let $\tau \in \Sigma, \tau^{\prime}=\sigma_{o} . \rho_{1} \cdots . \rho_{k}$ of type II'. If $\sigma_{o} \cap H$ is bounded, we have $\tau^{\prime}=\operatorname{pos}\left(\left(\sigma_{o} \cap H\right) \cup\left\{q_{1}, \ldots, q_{k}\right\}\right)$, and by Lemma 1.3, (c) $\tau^{\prime} \cap|\Sigma|=\sigma_{o}$ for sufficiently small $\varepsilon_{a}$, so $\tau \cap \tau^{\prime}=\tau \cap \sigma_{o}$ is a common face of $\tau$ and $\tau^{\prime}$.

If $\sigma_{o} \cap H$ is unbounded, we consider an affine hyperplane $H^{\prime}$ which does not contain 0 such that $\sigma_{o} \cap H^{\prime}$ is nonempty and bounded $\left(H^{\prime}\right.$ exists since 0 is the apex of $\left.\sigma_{o}\right)$. For sufficiently small $\varepsilon_{a}$ the cones $\rho_{1}, \ldots, \rho_{k}$ intersect $H^{\prime}$ in points $q_{1}^{\prime}, \ldots, q_{k}^{\prime}$, respectively, so 
that $\tau^{\prime}=\operatorname{pos}\left(\left(\sigma_{o} \cap H^{\prime}\right) \cup\left\{q_{1}^{\prime}, \ldots, q_{k}^{\prime}\right\}\right)$. We may again apply the arguments of Lemma 1.3, (c) so as to obtain $\tau^{\prime} \cap|\Sigma|=\sigma_{o}$ for sufficiently small $\varepsilon_{a}$.

For all the other choices of $\tau, \tau^{\prime}$ analogous arguments apply. Since only finitely many restrictions are imposed on $\varepsilon_{a}$, Lemma 1.4 follows.

Now we apply to $\Sigma^{(1)}$ the same procedure of extension as we applied to $\Sigma$, choosing as $\rho$ a 1-cone $\rho_{1}$ of the "old" fan $\Sigma$ (if there is a $\rho_{1}$ other than $\rho$ for which $\Sigma / \rho_{1}$ is incomplete). We denote the new fan by $\Sigma^{(2)}$. Continuing in this way, we find after a finite number $i$ of steps a fan $\Sigma^{(i)}=: \Sigma^{0}$ such that $\Sigma^{0} / \rho_{j}$ is complete for all 1-cones $\rho_{j}=\rho_{1}, \ldots, \rho_{i}$. We assert:

LEMmA 1.5. Given $\varepsilon>0, \Sigma^{0}$ can be chosen so that (2), (4) and (5) in Theorem 1.2 are satisfied for $\Sigma^{0}$ instead of $\Sigma_{0}$.

Proof. First we show that (2) in Theorem 1.2 holds for $\Sigma^{(1)}$ instead of $\Sigma^{0}$. In fact, by the definition of $\phi_{a}$ the points $p_{a, 1}, \ldots, p_{a, k}$ in II and the points $p_{1}, \ldots, p_{r}$ in III lie on the boundary of $|A|$ ( $A$ as in Lemma 1.3). Hence, by the definition of $\psi_{a}$, we see that $\rho_{a, 1}, \ldots, \rho_{a, k}\left(\right.$ see $\left.\mathrm{II}^{\prime}\right)$ and $\rho^{1}, \ldots, \rho^{r}$ (see III') lie in bd $\Sigma^{(1)}$. So if $\sigma$ is an element of $\Sigma^{(1)}$, we find $\sigma=\sigma_{0} \cdot \tau_{0}$, where $\sigma_{0} \in \Sigma$ and $\tau_{0}$ either equal some $\rho_{1}, \cdots . \rho_{k}$ (if $\sigma_{0}$ is different from $\rho_{1}$ ) or some $\rho^{1} \ldots . \rho^{r}$ (if $\sigma_{0}=\rho_{1}$ ). In both cases $\tau_{0}$ is a simplicial cone in bd $\Sigma^{(1)}$.

According to the construction of $\Sigma^{(2)}$ we see, analogously, that for $\sigma \in \Sigma^{(2)} \backslash \Sigma^{(1)}$ we have $\sigma=\sigma_{1} \cdot \tau_{1}$, where $\sigma_{1} \in \Sigma^{(1)}$ and $\tau_{1}$ is a simplicial cone in bd $\Sigma^{(2)}$. Since either $\sigma_{1} \in \Sigma$ or $\sigma_{1}=\sigma_{0} \cdot \tau_{0}$ for $\sigma_{0} \in \Sigma$ and $\tau_{0}$ is a simplicial cone in bd $\Sigma^{(1)}$, we obtain $\sigma=\sigma_{0} \cdot \tau_{0} \cdot \tau_{1}$. According to Lemma 1.4 applied to $\Sigma^{(1)}, \Sigma^{(2)}$ instead of $\Sigma, \Sigma^{(1)}$, respectively, the choice of a sufficiently small $\varepsilon_{a, 1}$ (instead of $\varepsilon_{a}$ ) guarantees that $\tau_{0} \in$ bd $\Sigma^{(2)}$. Therefore, $\tau_{0} . \tau_{1} \in$ bd $\Sigma^{(2)}$ so that (2) holds for $\Sigma, \Sigma^{(2)}$ instead of $\Sigma, \Sigma_{0}$, respectively. Continuing in this way we find that (2) is also satisfied if we replace $\Sigma^{(2)}$ successively by $\Sigma^{(3)}, \ldots, \Sigma^{(i)}=\Sigma^{0}$. Let hereby $\varepsilon_{a, 2}, \ldots, \varepsilon_{a, i}$ replace $\varepsilon_{a, 1}$.

(4) is readily implied by the construction of $\Sigma^{0}$. In order to obtain (5) we choose $\varepsilon_{a, 1}, \ldots, \varepsilon_{a, i}$ all to be smaller than the given $\varepsilon>0$. Since $p_{a, 1}, \ldots, p_{a, k}$ in II, $p^{1}, \ldots, p^{r}$ in III, and their analogs in the constructions of $\Sigma^{(2)}, \ldots, \Sigma^{(i)}$ all lie in the $\varepsilon$-neighborhood of $\Sigma$, the same is true for $\tau_{0}, \tau_{1}$ and their analogs in $\Sigma^{(2)}, \ldots, \Sigma^{(i)}=\Sigma^{0}$. This implies (5) for $\Sigma^{0}$.

If $\left|\Sigma^{0}\right|$ is contained in a (linear closed) half-space, we add to $\Sigma^{0}$ an $n$-dimensional simplicial cone $\sigma$ and its faces so that $\sigma \cap\left|\Sigma^{0}\right|=\{0\}$ and $\operatorname{pos}\left(\sigma \cup\left|\Sigma^{0}\right|\right)=\boldsymbol{R}^{n}$. The extended fan we denote again by $\Sigma^{0}$.

In order to construct $\Sigma_{0}$ and $\Sigma^{\prime}$ we consider the set $P=\operatorname{cl}\left(\boldsymbol{R}^{n} \backslash\left|\Sigma^{0}\right|\right) . P$ and $\left|\Sigma^{0}\right|$ have a common boundary which carries a subfan $\Sigma^{00}$ of $\Sigma^{0}$ and is a union $F_{1} \cup \cdots \cup F_{m}$ of $(n-1)$-dimensional cones of $\Sigma^{00}$. Let

$$
H_{i}:=\operatorname{lin} F_{i}, \quad i=1, \ldots, m
$$

be the linear hyperplanes spanned by $F_{i}$, and let $H_{i}^{+}, H_{i}^{-}$be the closed half-spaces bounded by $H_{i}$. 
LEMMA 1.6. $\quad \Sigma^{0}$ can be mapped isomorphically onto a fan in such a way that $|\Sigma|$ remains pointwise fixed, and that all $H_{i}$ are different. We denote the new fan again by $\Sigma^{0}$.

Proof. Each cone in $\Sigma^{0} \backslash \Sigma$ is either a multifold pyramid with basis in $\Sigma$ and apex-1cones not in $\Sigma$ or a simplicial cone with generating 1-cones not in $\Sigma$. Replacing the 1-cones by 1-cones in sufficiently small neighborhoods does not change the structure of $\Sigma^{0}$ and leaves $\Sigma$ unchanged. The new 1-cones may be chosen so that no two of the hyperplanes $H_{1}, \ldots, H_{m}$ coincide. This proves Lemma 1.6.

$H_{1}, \ldots, H_{m}$ split $\boldsymbol{R}^{n}$ into a system $M$ of polyhedral $n$-cones with apex 0 , each of which is an intersection of half-spaces $H_{i}^{+}, H_{i}^{-}$.

LEMMA 1.7. If $\sigma \in M$, then $\sigma$ is either totally contained in $P$ or in $\left|\Sigma^{0}\right|$.

Proof. Suppose $\sigma$ contains a point $x \in$ int $P$ and a point $y \in$ int $\left|\Sigma^{0}\right|$. Then we assert: The line segment $[x, y]$ intersects at least one $F_{i}, i=1, \ldots, m$. Indeed, this follows from a generalized version of the Jordan Curve Theorem; but we can see it directly: $[x, y]$ intersects finitely many $n$-cones of $\Sigma^{0}$. Among these the one closest to $x$ contains the point $z \in[x, y] \cap\left|\Sigma^{0}\right|$ closest to $x$ on its boundary. Since the boundary of $\left|\Sigma^{0}\right|$ is covered by $F_{1}, \ldots, F_{m}, z$ lies on one of the $F_{i}$. Then $H_{i}$ separates $x$ and $y$, a contradiction.

By Lemma 1.7, $P$ is the union of the cones of a subset $M_{0} \subset M$. Although $\boldsymbol{R}^{n}$ is covered by $\Sigma^{0}$ and $M_{0}$, the union of $\Sigma^{0}, M_{0}$ and the faces of the cones of $M_{0}$ do not, in general, provide a fan, since the common boundary of $\left|\Sigma^{0}\right|$ and $P$ is covered differently by cones of $\Sigma^{0}$ and faces of cones of $M_{0}$. However, Lemma 1.6 and Lemma 1.7 imply:

LEMMA 1.8. If a face $\mu$ of a cone of $M_{0}$ is contained in $\left|\Sigma^{0}\right|$, it is contained in an $F_{i}$, $i \in\{1, \ldots, m\}$.

So each $F_{i}$ is the union of the $(n-1)$-faces of cones of $M_{0}$. This remains true if we refine $M_{0}$ as follows.

LEMMA 1.9. The fan $\Sigma\left(M_{0}\right)$ consisting of $M_{0}$ and the faces of $M_{0}$ may be turned into a simplicial fan $\Sigma_{1}$ having the same 1-cones as $\Sigma\left(M_{0}\right)$ by splitting the cones of $\Sigma\left(M_{0}\right)$.

PROOF. This follows from a combinatorial theorem (see [E, III, Theorem 2.6]).

Now we adjust $\Sigma^{0}$ to $\Sigma$ as follows. Each cone $\sigma \in \Sigma^{0} \backslash \Sigma$ which is not contained in the boundary of $\left|\Sigma\left(M_{0}\right)\right|$ is a $k$-fold pyramid $\tau . \rho_{1}, \cdots . \rho_{k}=\tau . \tau^{\prime}$, where $\tau^{\prime}=\rho_{1}, \cdots . \rho_{k}$ is a simplicial cone and the basis $\tau$ lies in $\Sigma^{0}$. In $\Sigma_{1}, \tau^{\prime}$ is split into simplicial cones $\tau_{1}, \ldots, \tau_{s}$, hence $\sigma$ is split into simplicial cones $\tau . \tau_{1}, \ldots, \tau$. $\tau_{s}$. This turns $\Sigma^{0}$ into a fan $\Sigma_{0}$.

LEMMA 1.10. Lemma 1.5 is true for $\Sigma_{0}$ instead of $\Sigma^{0}$.

PROOF. This readily follows from $\left|\Sigma^{0}\right|=\left|\Sigma_{0}\right|$ and the construction of $\Sigma_{0}$.

The fan $\Sigma_{0} \cup M_{0}$ is complete; we denote it by $\Sigma^{\prime}$. So $\Sigma_{0}, \Sigma^{\prime}$ satisfy all the properties (1) through (5) and the proof of Theorem 1.2, hence also the proof of Theorem 1.1, is completed. 
2. The Zariski-Riemann space of a rational fan. Let $r \geq 0$ be an integer, and $N$ a free $\boldsymbol{Z}$-module of rank $r . N_{\boldsymbol{R}}:=N \otimes_{\boldsymbol{Z}} \boldsymbol{R}$ is an $r$-dimensional real space with the lattice $N$. We consider cones and fans in $N_{\boldsymbol{R}}$ from this section on. Namely, a subset $C \subset N_{\boldsymbol{R}}:=N \otimes \boldsymbol{R}$ is said to be a convex polyhedral cone if there exists a finite subset $\left\{y_{1}, \ldots, y_{s}\right\} \subset N_{\boldsymbol{R}}$ with

$$
C=\operatorname{pos}\left\{y_{1}, \ldots, y_{s}\right\}=\boldsymbol{R}_{0} y_{1}+\cdots+\boldsymbol{R}_{0} y_{s}
$$

where $\boldsymbol{R}_{0}$ is the set of nonnegative real numbers. The cone $C$ is said to be rational if we can choose $y_{1}, \ldots, y_{s}$ in $N$, and strongly convex if $C \cap(-C)=\{0\}$.

Let $M:=\operatorname{Hom}_{\boldsymbol{Z}}(N, \boldsymbol{Z})$ and $M_{\boldsymbol{R}}:=M \otimes \boldsymbol{R}$. There exists a natural perfect pairing $\langle\rangle:, M_{\boldsymbol{R}} \times N_{\boldsymbol{R}} \rightarrow \boldsymbol{R}$. A subset $C^{\prime} \subset C$ is a face and written $C^{\prime} \prec C$ if there exists $x \in M_{\boldsymbol{R}}$ with $C \subset(x \geq 0)$ and $C^{\prime}=C \cap(x=0)$, where we denote $(x \geq 0)=\left\{y \in N_{\boldsymbol{R}} ;\langle x, y\rangle \geq 0\right\}$ and $(x=0)=\left\{y \in N_{\boldsymbol{R}} ;\langle x, y\rangle=0\right\}$.

From this section on, we prefer to use letters $X, Y, \ldots$ for fans rather than Greek capitals. Recall that a nonempty set $X$ of strongly convex rational polyhedral cones is said to be a fan if

(i) $\sigma \in X$ and $\eta \prec \sigma$ imply $\eta \in X$, and

(ii) if $\sigma, \tau \in X$, then $\sigma \cap \tau$ is a common face of $\sigma$ and $\tau$.

The condition (ii) can be replaced by the following "separability condition" (ii') (cf. [F2, 1.2, (12)]).

(ii') For $\sigma, \tau \in X$, there exists $x \in M_{\boldsymbol{R}}$ with $\sigma \subset(x \geq 0), \tau \subset(x \leq 0)$ and $\sigma \cap(x=0)=\tau \cap(x=0)$.

The set $F(\pi)$ of all faces of a strongly convex rational polyhedral cone $\pi$ is a fan with the unique maximal element $\pi$. Such a fan is called an affine fan.

For each $\sigma \in X$, the dual cone $\sigma^{\vee} \subset M_{\boldsymbol{R}}$ is defined by

$$
\sigma^{\vee}:=\left\{x \in M_{R} ;\langle x, u\rangle \geq 0 \text { for any } u \in \sigma\right\} .
$$

This is an $r$-dimensional polyhedral cone. Since $M \cap \sigma^{\vee}$ is a finitely generated additive semigroup (cf. [01, Prop.1.1]), the semigroup ring $\boldsymbol{C}\left[M \cap \sigma^{\vee}\right]$ over the complex number field is an affine ring and the quotient field is equal to that of the group ring $C[M]$. The toric variety $X_{C}$ associated to a fan $X$ is defined to be the union of the affine toric varieties Spec $C\left[M \cap \sigma^{\vee}\right]$ for $\sigma \in X$. The function field of $X_{C}$ is the quotient field of $C[M]$, and the algebraic torus $T_{N}:=\operatorname{Spec} C[M]$ acts on $X_{C}$. The toric variety $X_{C}$ is of finite type, i.e., it is an algebraic variety if and only if the fan $X$ is finite.

The topology of a fan $X$ is defined as follows. A subset $U \subset X$ is defined to be open if $\sigma \in U$ and $\eta \prec \sigma$ imply $\eta \in U$. Namely, $U$ is open if and only if it is empty or a subfan of $X$. For each point $x \in X_{\boldsymbol{C}}$, we define $\phi(x)$ to be the minimal $\sigma \in X$ with $x \in \operatorname{Spec} \boldsymbol{C}\left[M \cap \sigma^{\vee}\right]$. Then the map $\phi: X_{C} \rightarrow X$ is continuous.

A collection of convex polyhedral cones not necessarily rational is called a real fan if it satisfies (i) and (ii). Usual fans which define toric varieties are sometimes called rational fans. 
The support $|X|$ of a fan $X$ is defined to be $\bigcup_{\sigma \in X} \sigma$, and $X$ is said to be complete if it is finite and $|X|=N_{\boldsymbol{R}}$. For a rational finite fan $X$, the toric variety $X_{\boldsymbol{C}}$ is complete if and only if $X$ is complete. If $X$ is a subfan of a complete rational fan $\bar{X}$, then $X_{C}$ is an open subvariety of $\bar{X}_{\boldsymbol{C}}$, i.e., $\bar{X}_{\boldsymbol{C}}$ is a completion of $X_{\boldsymbol{C}}$.

A subring $R$ of a field $K$ is said to be a valuation ring if it satisfies the condition:

$$
1 / x \in R \text { for any } x \in K \backslash R \text {. }
$$

In particular, $R=K$ is a valuation ring.

Let $(R, \mathfrak{m})$ and $\left(R^{\prime}, \mathfrak{m}^{\prime}\right)$ be not necessarily Noetherian local rings with a common quotient field $K$. We say $R^{\prime}$ dominates $R$ and write $R \leq R^{\prime}$ if $R \subset R^{\prime}$ and $\mathfrak{m}=\mathfrak{m}^{\prime} \cap R$.

It is known that, for any local ring $(R, \mathfrak{m})$ with the quotient ring $K$, there exists a valuation ring $\left(R^{\prime}, \mathfrak{m}^{\prime}\right)$ which dominates $(R, \mathfrak{m})$. When $(R, \mathfrak{m})$ is a local ring of an algebraic variety, $R^{\prime}$ can be regarded as the limit of a transfinite sequence of blowups of $R$.

Let $X$ be an algebraic variety over a field $k$, i.e., a reduced and irreducible separated scheme of finite type over $k$, and $K$ the function field. We say a valuation ring $R$ of $K$ dominates a point $x$ of the scheme $X$ if it dominates the local ring $\mathcal{O}_{x}$. We denote by $\mathrm{ZR}(X)$ the set of all valuation rings of $K$ which dominates a point of $X$ (cf. [ZS, Chap. VI, §17]). $\mathrm{ZR}(X)$ is called the Zariski-Riemann space of $X$. We denote by $\mathrm{ZR}(K)$ the set of all valuation rings of $K$ which contains the base field $k$.

The following theorem is known as the valuative criterion of properness of varieties (cf. [H, Thm. 4.7]).

THEOREM 2.1. An algebraic variety $X$ with the function field $K$ is complete if and only if $\mathrm{ZR}(X)=\mathrm{ZR}(K)$.

For an algebraic variety $X$, the topology of $\mathrm{ZR}(X)$ is defined as follows (cf. [N1, §1]). For a proper birational morphism $X^{\prime} \rightarrow X$ and a closed subset $Y^{\prime} \subset X^{\prime}$, let $F$ be the set of all valuation rings in $\mathrm{ZR}(X)$ which dominate points of $Y^{\prime}$. We define the set of all such $F$ as a basis of the closed sets of $\mathrm{ZR}(X)$. This topology is equal to the topology defined by the open basis consisting of the following $E(B)$ 's. Let $B$ be an integral domain of finite type over $k$ with the quotient field $K$, and $E(B)$ the set of all valuation rings in $\mathrm{ZR}(X)$ which contain $B$.

The following theorem was used in Nagata's compactification theorem.

TheOrem 2.2 ([ZS, Thm. 40], [N1, Prop. 1.1]). The space $\mathrm{ZR}(X)$ is quasi-compact for any algebraic variety $X$.

We define the Zariski-Riemann space for a rational fan. In this case, we replace the field $K$ by the dual module $M \simeq Z^{r}$ of $N$.

A relation $\leq$ on $M$ is said to be an additive preorder if it satisfies the following conditions:

(1) For any $x, y \in M$, either $x \leq y$ or $y \leq x$ is satisfied.

(2) $x \leq y$ and $y \leq z$ imply $x \leq z$.

(3) If $x \leq y$, then $x+z \leq y+z$ for every $z \in M$. 
Note that we do not assume the anti-symmetry: $x \leq y$ and $y \leq x$ imply $x=y$. It is easy to see that $x \leq y$ and $x^{\prime} \leq y^{\prime}$ imply $x+x^{\prime} \leq y+y^{\prime}$, and $0 \leq n x$ for an integer $n>0$ implies $0 \leq x$.

Definition 2.3. We define the Zariski-Riemann space $\mathrm{ZR}(M)$ to be the set of all additive preorders of $M$.

We are going to define the topology of $\mathrm{ZR}(M)$. Each element $v$ of $\mathrm{ZR}(M)$ is denoted by $\leq_{v}$ when it is used as a relational operator. We denote $x={ }_{v} y$ if $x \leq_{v} y$ and $y \leq_{v} x$, while $x<_{v} y$ if $x \leq_{v} y$ and not $y \leq_{v} x . L(v):=\left\{x \in M ; 0 \leq_{v} x\right\}$ is a substitute for the valuation ring in our case. We see easily that $L(v)$ is a subsemigroup satisfying $-x \in L(v)$ for all $x \in M \backslash L(v)$. We define $L^{0}(v):=\left\{x \in M ; x={ }_{v} 0\right\}$, which is equal to the $Z$-submodule $L(v) \cap(-L(v))$ of $M . M / L^{0}(v)$ is equal to the quotient of $M$ by the equivalence relation $x={ }_{v} y$, and is a free $Z$-module.

We denote by $\eta(M)$ the trivial preorder of $M$, i.e., $L^{0}(\eta(M))=M$.

$$
\phi_{M}: \operatorname{ZR}(M) \backslash\{\eta(M)\} \rightarrow\left(N_{\boldsymbol{R}} \backslash\{0\}\right) / \boldsymbol{R}_{+}
$$

is defined as follows. For $v \in \mathrm{ZR}(M) \backslash\{\eta(M)\}$, let $C_{v}$ be the convex closure of $L(v)$ in $M_{\boldsymbol{R}}$. Then the closure $\bar{C}_{v}$ is a closed half space (cf. Lemma 5.1). Hence there exists $x \in N_{\boldsymbol{R}} \backslash\{0\}$ with $\bar{C}_{v}=(x \geq 0)$. We define $\phi_{M}(v)$ to be the image of $x$ in $\left(N_{\boldsymbol{R}} \backslash\{0\}\right) / \boldsymbol{R}_{+}$.

We set $S_{N}:=\left(N_{\boldsymbol{R}} \backslash\{0\}\right) / \boldsymbol{R}_{+}$. Then $\mathrm{ZR}(M)$ has a recursive structure as follows. For each $x \in N_{\boldsymbol{R}} \backslash\{0\}$, let $\bar{x}$ be its image in $S_{N}$. Let $M(x)_{\boldsymbol{R}}$ be the largest rational subspace of $M_{\boldsymbol{R}}$ contained in $(x=0)$, and let $M(x):=M(x)_{\boldsymbol{R}} \cap M$. Then $\phi_{M}^{-1}(\bar{x})$ is identified with $\mathrm{ZR}(M(x))$ by identifying each $v \in \phi_{M}^{-1}(\bar{x})$ with its restriction to $M(x)$.

Let $C$ be a rational polyhedral cone in $N_{R}$ which is not necessarily strongly convex. Then there exists a finite subset $\left\{x_{1}, \ldots, x_{s}\right\} \subset M$ with

$$
C=\left(x_{1} \geq 0\right) \cap \cdots \cap\left(x_{s} \geq 0\right)
$$

(cf. [O1, Thm. A.2]). We define a subset $\|C\|$ of $\mathrm{ZR}(M)$ by

$$
\begin{aligned}
\|C\| & :=\left\{v \in \operatorname{ZR}(M) ; 0 \leq v x_{i}, i=1, \ldots, s\right\} \\
& =\left\{v \in \operatorname{ZR}(M) ; M \cap C^{\vee} \subset L(v)\right\} .
\end{aligned}
$$

For any rational polyhedral cones $C_{1}, C_{2}$, we see easily that $M \cap C_{1}^{\vee}, M \cap C_{2}^{\vee} \subset L(v)$ implies $M \cap\left(C_{1}^{\vee}+C_{2}^{\vee}\right) \subset L(v)$. Since $C_{1}^{\vee}+C_{2}^{\vee}=\left(C_{1} \cap C_{2}\right)^{\vee}$, we have $\left\|C_{1}\right\| \cap\left\|C_{2}\right\|=\left\|C_{1} \cap C_{2}\right\|$. We take the set of all such $\|C\|$ 's as the open basis of $\mathrm{ZR}(M)$. Since the set of all finite subsets of $M$ is countable, the topology of $\mathrm{ZR}(M)$ defined by this open basis satisfies the second countability axiom.

Let $\mathrm{ZR}_{0}(M)$ be the set of the elements in $\mathrm{ZR}(M)$ which have the anti-symmetry property, i.e., the set of additive orders of $M$. If we identify $M$ with the set of monomials of a Laurent polynomial ring, $\mathrm{ZR}_{0}(M)$ is equal to the space introduced by Kuroda [K] (see also [S3]). Kuroda $[\mathrm{K}]$ introduced this space in order to prove the infinity of the SAGBI bases of some invariant rings.

We omit the proof of the following proposition which we do not use in this paper. 
Proposition 2.4. $\mathrm{ZR}_{0}(M)$ is closed in $\mathrm{ZR}(M)$ and the induced topology of $\mathrm{ZR}_{0}(M)$ is equal to that of Kuroda.

The Zariski-Riemann space $\mathrm{ZR}(X)$ of a fan $X$ is defined by

$$
\mathrm{ZR}(X):=\bigcup_{\sigma \in \Delta}\|\sigma\|
$$

THEOREM 2.5. The Zariski-Riemann space $\mathrm{ZR}(M)$ is quasi-compact. $\mathrm{ZR}(X)$ is quasicompact for any finite fan X of $M_{\boldsymbol{R}}$. Here "quasi-compact" means "compact but not necessarily Hausdorff".

Proof. We follow the method of Zariski-Samuel [ZS, Thm. 40]. For every $m \in M \backslash\{0\}$, we set $\hat{S}_{m}^{0}:=\{-1,0,1\}$. For $v \in \mathrm{ZR}(M)$ and $m \in M$, we define $v(m) \in \hat{S}_{m}^{0}$ to be -1 if $m<{ }_{v} 0,0$ if $m={ }_{v} 0$ and 1 if $0<_{v} m$. Since $v \in \mathrm{ZR}(M)$ is determined by the set of $m$ 's with $0 \leq_{v} m$, we regard $v$ as the map from $M$ to $\{-1,0,1\}$, and we get an embedding

$$
\mathrm{ZR}(M) \subset \prod_{m \in M \backslash\{0\}} \hat{S}_{m}^{0} .
$$

The weak topology of $\hat{S}_{m}^{0}$ is defined by setting $\{\varnothing,\{0\},\{0,1\},\{-1,0,1\}\}$ as the set of open subsets. Since the set of finite intersections of $\{v ; v(m)=0,1\}$ is an open basis of $\mathrm{ZR}(M)$, the topology of $\mathrm{ZR}(M)$ is equal to the relative topology of the product topology of $\prod_{m \in M} \hat{S}_{m}^{0}$. Now we introduce the discrete topology on $\hat{S}_{m}^{0}$. Then the product space is compact by Tychonoff's theorem. $\mathrm{ZR}(M)$ is a closed subset of the compact product space. Actually, it is defined by the equalities

$$
\begin{gathered}
v(m)=-1 \quad \text { or } \quad v\left(m^{\prime}\right)=-1 \quad \text { or } \quad v\left(m+m^{\prime}\right)=0,1, \\
v(m)=0,1 \quad \text { or } \quad v(-m)=1
\end{gathered}
$$

and

$$
v(m)=0,-1 \quad \text { or } \quad v(-m)=-1
$$

for all $m, m^{\prime} \in M$. Hence $\mathrm{ZR}(M)$ is compact in the strong topology, hence so is it in the weak topology.

In order to show the compactness of $\mathrm{ZR}(X)$ for a finite fan $X$, it suffices to show that of each $\|\sigma\|$. We can show the compactness of $\|\sigma\|$ by taking a generator $\left\{x_{1}, \ldots, x_{s}\right\} \subset M$ of the cone $\sigma^{\vee}$ and adding the equalities

$$
v\left(x_{i}\right)=0,1 \quad(i=1, \ldots, s)
$$

to those of (1), (2) and (3).

Let $v$ be an element of $\operatorname{ZR}(M)$. We will express $v$ by a sequence of elements in $N_{\boldsymbol{R}}$. We set $v_{0}:=v$ and $M^{0}(v):=M$. If $v_{0} \neq \eta\left(M^{0}(v)\right)$, then let $M^{1}(v)$ be the intersection $M \cap\left(x_{0}=0\right) \subset M_{R}$ for $x_{0}:=\phi_{M}\left(v_{0}\right)$, and $v_{1}$ the restriction of $v_{0}$ to $M^{1}(v)$. Inductively, if $v_{i-1} \neq \eta\left(M^{i-1}(v)\right)$, we define $M^{i}(v)$ to be the intersection $M^{i-1}(v) \cap\left(x_{i-1}=0\right) \subset$ $M^{i-1}(v)_{\boldsymbol{R}}$ for $x_{i-1}:=\phi_{M^{i-1}(v)}\left(v_{i-1}\right)$, and $v_{i}$ the restriction of $v_{i-1}$ to $M^{i}(v)$. Since the rank 
of $M$ is $r$, there exists a nonnegative integer $s \leq r$ with $v_{s}=\eta\left(M^{s}(v)\right)$. We call $s$ the rank of $v$ and denote it by $\operatorname{rank}(v)$. The rank of $\eta(M)$ is defined to be 0 . This is an analog of the rank defined for a valuation ring.

Note that the preorder $v$ is recovered from the integer $s$ and the sequence $\left(x_{0}, \ldots, x_{s-1}\right)$. Actually, if we take a representative $y_{i} \in N_{\boldsymbol{R}}$ of $x_{i}$ for each $i$, then $m \in L(v)$ if and only if either there exists $0 \leq j \leq s-1$ such that $\left\langle m, y_{k}\right\rangle=0$ for $0 \leq k<j$ and $\left\langle m, y_{j}\right\rangle>0$, or $\left\langle m, y_{k}\right\rangle=0$ for all $0 \leq k \leq s-1$. This is also equivalent to the condition $\left\langle m, \sum_{i=0}^{s-1} \varepsilon^{i} y_{i}\right\rangle \geq 0$ for a sufficiently small positive real number $\varepsilon$. Since we often use this sequence $\left(y_{0}, \ldots, y_{s-1}\right)$ of points in $N_{\boldsymbol{R}}$, we call it a defining sequence of $v$. By construction, $y_{0}, \ldots, y_{j-1}$ are 0 on $M^{j}(v)_{\boldsymbol{R}}$ and $y_{j}$ is not identically 0 on this linear subspace. Since $\left\{M^{j}(v)_{\boldsymbol{R}}\right\}$ is a shrinking sequence of vector spaces, $\left\{y_{0}, \ldots, y_{s-1}\right\}$ is linearly independent.

When $v \neq \eta(M)$, the first generalization $v^{\prime} \in \mathrm{ZR}(M)$ of $v$ is defined by

$$
x \leq_{v^{\prime}} y \Longleftrightarrow x \leq_{v} y \text { or } y-x \in M^{\operatorname{rank}(v)-1}(v) .
$$

If $\operatorname{rank}(v)=s$ and $\left(y_{0}, \ldots, y_{s-1}\right)$ is a defining sequence of $v$, then $\left(y_{0}, \ldots, y_{s-2}\right)$ is a defining sequence of $v^{\prime}$. Hence, we get $\eta(M)$ by $s$-times repetition of the first generalization starting from $v$. For $v, w \in \mathrm{ZR}(M), w$ is said to be a generalization of $v$ if $L(v) \subset L(w)$. This is equivalent to the condition that we get $w$ from $v$ by a finite repetition of the first generalization.

If $\operatorname{rank}(v)=s$, then $M^{s}(v)$ is equal to the $Z$-submodule $L^{0}(v)$ of $M$. We say that an element $v$ of $\mathrm{ZR}(M)$ dominates a cone $C$ of $N_{\boldsymbol{R}}$ if $M \cap C^{\vee} \subset L(v)$ and $M \cap C^{\vee} \cap L^{0}(v)=$ $M \cap C^{\perp}$, where $C^{\perp}:=\left\{x \in M_{\boldsymbol{R}} ;\langle x, y\rangle=0\right.$ for all $\left.y \in C\right\}$. This situation is described by the sequence $\left(M^{0}(v), \ldots, M^{s}(v)\right)$ and the defining sequence $\left(y_{0}, \ldots, y_{s-1}\right)$ of $v$ as follows. The additive preorder $v$ dominates $C$ if and only if

$$
M^{i}(v) \cap C^{\vee} \subset\left(y_{i} \geq 0\right)
$$

for each $i=0, \ldots, s-1$, and

$$
M^{s}(v) \cap C^{\vee}=M \cap C^{\perp} .
$$

LEMMA 2.6. Let $\left(y_{0}, \ldots, y_{s-1}\right)$ be a defining sequence of $v \in \mathrm{ZR}(M)$. For a positive real number $\varepsilon$, we set $z_{\varepsilon}:=\sum_{i=0}^{s-1} \varepsilon^{i} y_{i}$. Then the following conditions on a rational polyhedral cone $C$ are equivalent.

(1) $v$ dominates $C$.

(2) There exists $\varepsilon_{0}>0$ such that $z_{\varepsilon} \in$ rel. int $C$ for every $0<\varepsilon \leq \varepsilon_{0}$.

(3) There exists a sequence $\left\{\varepsilon_{j}\right\}$ of positive real numbers with $\lim _{j \rightarrow \infty} \varepsilon_{j}=0$ and $z_{\varepsilon_{j}} \in$ rel. int $C$ for every $j$.

Proof. We show (1) $\Rightarrow$ (2). We take $m_{1}, \ldots, m_{t} \in M \cap\left(C^{\vee} \backslash C^{\perp}\right)$ and $m_{t+1}, \ldots, m_{u} \in$ $M \cap C^{\perp}$ so that they generate the semigroup $M \cap C^{\vee}$. Since $C^{\perp}$ is a rational subspace, it is generated by $\left\{m_{t+1}, \ldots, m_{u}\right\}$. For each $m_{i}$ with $1 \leq i \leq t$, there exists $1 \leq j \leq s-1$ such that

$$
\left\langle m_{i}, y_{0}\right\rangle=\cdots=\left\langle m_{i}, y_{j-1}\right\rangle=0, \quad\left\langle m_{i}, y_{j}\right\rangle>0
$$


Hence, $\left\langle m_{i}, z_{\varepsilon}\right\rangle>0$ for a sufficiently small $\varepsilon>0$ for $i=1, \ldots, t$. Since $\left\langle m_{i}, z_{\varepsilon}\right\rangle=0$ for every $t+1 \leq i \leq u$, we have $C^{\vee} \subset\left(z_{\varepsilon} \geq 0\right)$ and $C^{\vee} \cap\left(z_{\varepsilon}=0\right)=C^{\perp}$ for such $\varepsilon$. Hence $z_{\varepsilon} \in \operatorname{rel}$. int $C$. (2) $\Rightarrow(3)$ is obvious.

We show (3) $\Rightarrow(1)$. The condition implies $M^{i}(v)_{\boldsymbol{R}} \cap C^{\vee} \subset\left(z_{\varepsilon_{j}} \geq 0\right)$ for $i=0, \ldots$, $s-1$. Since $y_{0}=\cdots=y_{i-1}=0$ on $M^{i}(v)_{R}$, the limit of the linear functions $\varepsilon_{j}^{-i} z_{\varepsilon_{j}}$ of $M^{i}(v)_{\boldsymbol{R}}$ is equal to $y_{i}$. Hence $M^{i}(v)_{\boldsymbol{R}} \cap C^{\vee} \subset\left(y_{i} \geq 0\right)$ for every $i$. This implies $M \cap C^{\vee} \subset$ $L(v)$. Let $\varepsilon:=\varepsilon_{1}$. Then clearly, $z_{\varepsilon}=0$ on $M^{s}(v)_{\boldsymbol{R}}$. Hence

$$
M \cap C^{\vee} \cap L^{0}(v) \subset M \cap C^{\vee} \cap\left(z_{\varepsilon}=0\right)=M \cap C^{\perp} .
$$

On the other hand, $C^{\perp} \subset\left(z_{\varepsilon_{j}}=0\right)$ for $j=1, \ldots, s$ imply that $y_{0}, \ldots, y_{s-1}$ are zero on $C^{\perp}$, and hence $M \cap C^{\perp} \subset L^{0}(v)$. Hence, $v$ dominates $C$.

The following proposition is an analog of the valuative criterion of separatedness of an algebraic prevariety.

Proposition 2.7. For a fan $X$ of $N_{\boldsymbol{R}}$, an element $v \in \mathrm{ZR}(M)$ dominates at most one cone of $X$. Conversely, if $X$ is a union of affine fans and any $v \in \mathrm{ZR}(M)$ dominates at most one cone of $X$, then $X$ is a fan.

ProOF. If $\sigma$ and $\tau$ are distinct cones of the fan $X$, then rel. int $\sigma \cap \operatorname{rel}$. int $\tau=\emptyset$. If $v$ dominates $\sigma$, then it does not dominates $\tau$ since the condition (2) of Lemma 2.6 is satisfied for $C=\sigma$

Now we prove the second part. It suffices to show that $\sigma \cap \tau$ is a face of $\sigma$ for any $\sigma, \tau \in X$. Any point $y$ in the relative interior of the cone $\sigma \cap \tau$ is contained in the relative interior of a face $\sigma_{1}$ of $\sigma$ and in that of a face $\tau_{1}$ of $\tau$. If we take $v \in \mathrm{ZR}(M)$ with $L(v)=$ $M \cap(y \geq 0)$, then $v$ dominates $\sigma_{1}$ and $\tau_{1}$, and hence $\sigma_{1}=\tau_{1}$ by assumption. By $\tau_{1} \subset \tau$, we have $\sigma_{1} \subset \sigma \cap \tau$. On the other hand, the defining element $x \in M_{\boldsymbol{R}}$ of the face $\sigma_{1} \subset \sigma$ defines a face of $\sigma \cap \tau$. This face is equal to $\sigma \cap \tau$ itself, since it contains $y$ in the relative interior. We know $\sigma \cap \tau \subset \sigma_{1}$, since $\sigma_{1}=\sigma \cap(x=0)$. Hence $\sigma \cap \tau=\sigma_{1}$, and $\sigma \cap \tau$ is a face of $\sigma$.

Definition 2.8. We denote by $\operatorname{dom}(\sigma)$ the set of elements of $\operatorname{ZR}(M)$ which dominate the strongly convex rational polyhedral cone $\sigma$.

If we take a point $y$ in a strongly convex rational polyhedral cone $\sigma$, then the rank one element $v \in \mathrm{ZR}(M)$ with $L(v)=M \cap(y \geq 0)$ dominates $\sigma$. In particular, $\operatorname{dom}(\sigma)$ is not empty.

LEMMA 2.9. Let $C$ be a rational polyhedral cone of $N_{R}$, and $v$ an element of $\mathrm{ZR}(M)$. Then $M \cap C^{\vee} \subset L(v)$ if and only if $v$ dominates a face of $C$. In particular, if $\pi$ is a strongly convex rational polyhedral cone, then we have the equality

$$
\|\pi\|=\operatorname{ZR}(F(\pi))=\bigcup_{\sigma \in F(\pi)} \operatorname{dom}(\sigma) .
$$


Proof. If $v$ dominates a face $C_{1}$ of $C$, then

$$
M \cap C^{\vee} \subset M \cap C_{1}^{\vee} \subset L(v) .
$$

Conversely, suppose $M \cap C^{\vee} \subset L(v)$. We see easily by induction that $C^{\vee} \cap M^{i}(v)_{\boldsymbol{R}}$ is a face of $C^{\vee}$ for $i=0, \ldots, \operatorname{rank}(v)$. In particular, $C^{\vee} \cap L^{0}(v)_{R}$ is a face of $C^{\vee}$. Hence there exists a face $C_{1} \prec C$ with $C^{\vee} \cap L^{0}(v)_{\boldsymbol{R}}=C^{\vee} \cap C_{1}^{\perp}$ (cf. [01, Prop. A.6]). Since $C_{1}^{\perp} \subset L^{0}(v)_{R}$ and $C_{1}^{\vee}=C^{\vee}+C_{1}^{\perp}$ by [O1, Cor. A.7], we have $M \cap C_{1}^{\vee} \subset L(v)$ and $M \cap C_{1}^{\vee} \cap L(v)=M \cap C_{1}^{\perp}$. Hence $v$ dominates $C_{1}$.

The equalities are now obvious.

LEMMA 2.10. Let $C$ be a rational polyhedral cone of $N_{\boldsymbol{R}}$, and $v$ an element of $\mathrm{ZR}(M)$ which dominates $C$. If $w$ is a generalization of $v$, then $w$ dominates a face of $C$. The dimension of $C$ is at least $\operatorname{rank}(v)$.

Proof. We have $L(v) \subset L(w)$ since $w$ is a generalization of $v$. Since $M \cap C^{\vee} \subset$ $L(w), w$ dominates a face $C^{\prime}$ of $C$ by Lemma 2.9. Let $\operatorname{rank}(v)=s$ and let $\left(y_{0}, \ldots, y_{s-1}\right)$ be a defining sequence of $v$. By Lemma 2.6, $z_{\varepsilon}:=\sum_{i=0}^{s-1} \varepsilon^{i} y_{i}$ satisfies $z_{\varepsilon} \in \operatorname{rel}$. int $C$ for a sufficiently small $\varepsilon>0$. Since $y_{0}, \ldots, y_{s-1}$ are linearly independent, $z_{\varepsilon}$ 's for $s$ distinct $\varepsilon$ 's are also linearly independent by Vandermonde's equality. Hence the dimension of $C$ is at least $s$.

REMARK 2.11. $C$ is also a face of itself. Hence $w$ might dominates $C$ in Lemma 2.10.

For a fan $X$, we define

$$
\mathrm{ZR}(X)^{1}:=\{v \in \mathrm{ZR}(X) ; \operatorname{rank}(v)=1\} .
$$

Proposition 2.12. For finite fans $X, Y$, the following conditions are equivalent.

(1) $\mathrm{ZR}(X) \subset \mathrm{ZR}(Y)$.

(2) $\mathrm{ZR}(X)^{1} \subset \mathrm{ZR}(Y)^{1}$.

(3) $|X| \subset|Y|$.

ProOF. $\quad(1) \Rightarrow(2)$ is obvious.

For (2) $\Rightarrow$ (3), let $x \in \sigma \in X$. Since $|Y|$ contains 0 , we assume $x \neq 0$. Let $v$ be the preorder of rank one with $L(v)=M \cap(x \geq 0)$. Since $M \cap \sigma^{\vee} \subset M \cap(x \geq 0)$, we have $v \in \mathrm{ZR}(X)^{1}$. Since $v \in \mathrm{ZR}(Y)^{1}$ by (2), we have $M \cap \tau^{\vee} \subset L(v)=M \cap(x \geq 0)$ for a rational cone $\tau \in Y$. Then $\tau^{\vee} \subset(x \geq 0)$ and $x \in \tau \subset|Y|$.

We show $(3) \Rightarrow(1)$. Suppose $v \in \mathrm{ZR}(X)$ dominates $\sigma \in X$. Let $\operatorname{rank}(v)=s$ and $\left(y_{0}, \ldots, y_{s-1}\right)$ a defining sequence of $v$. Then by Lemma $2.6, z_{\varepsilon}:=\sum_{i=0}^{s-1} \varepsilon^{i} y_{i} \in$ rel. int $\sigma$ for sufficiently small $\varepsilon>0$. Since $z_{\varepsilon} \in|X| \subset|Y|$ and $Y$ is finite, there exist $\tau \in Y$ and a convergent sequence $\left\{\varepsilon_{j}\right\}$ with the limit 0 and $z_{\varepsilon_{j}} \in \operatorname{rel}$. int $\tau$. Hence $v \in \operatorname{dom} \tau \subset \operatorname{ZR}(Y)$ by Lemma 2.6.

Since the proposition is also true even if we exchange $X$ and $Y$, we have the following corollary.

COROLlaRY 2.13. For finite fans $X, Y$, the following conditions are equivalent. 
(1) $\operatorname{ZR}(X)=\operatorname{ZR}(Y)$.

(2) $\mathrm{ZR}(X)^{1}=\mathrm{ZR}(Y)^{1}$.

(3) $|X|=|Y|$.

3. Blowups of fans. Fans in this section are not necessarily rational and cones are finitely generated convex polyhedral cones unless otherwise mentioned.

Let $D$ be a cone of $M_{R}$. A nonempty convex subset $P$ of $M_{R}$ is said to be $D$-convex if it has the $D$-ideal property, i.e., if

$$
x \in P, y \in D \Rightarrow x+y \in P .
$$

For a subset $S$ of $M_{R}$, we set

$$
S^{\vee}:=\left\{y \in N_{\boldsymbol{R}} ;\langle x, y\rangle \geq 0 \text { for all } x \in S\right\} .
$$

For the convex hull $\operatorname{conv}(S)$ and the convex cone $\operatorname{Cone}(S)$ generated by $S$, we see easily the equalities

$$
S^{\vee}=\operatorname{conv}(S)^{\vee}=\operatorname{Cone}(S)^{\vee} .
$$

Here Cone $(S)$ is not necessarily finitely generated if $S$ is not a finite set. For subsets $S, T$ of $M_{R}$, clearly we have

$$
(S \cup T)^{\vee}=S^{\vee} \cap T^{\vee} .
$$

For a cone $C$, a $C^{\vee}$-convex set $P$ generated by a finite set $S$ is called a $C^{\vee}$-convex polyhedron. When $C$ is rational, $P$ is said to be rational if $S$ consists of finite rational points.

Let $C$ be a cone of $N_{\boldsymbol{R}}$. For an $r$-dimensional $C^{\vee}$-convex polyhedron $P$,

$$
\operatorname{Fan}(P):=\left\{(P-x)^{\vee} ; x \in P\right\}
$$

is a finite real fan with support $C$. If $P$ is rational, $\operatorname{Fan}(P)$ is also a rational fan. As is wellknown, the relationship between $P$ and $\operatorname{Fan}(P)$ is as follows. A subset $Q \subset P$ is called a face of $P$ if there exist an element $u \in N_{\boldsymbol{R}}$ and a real number $a$ with $P \subset(u \geq a)$ and $Q=P \cap(u=a)$, where $(u \geq a)=\left\{x \in M_{\boldsymbol{R}} ;\langle x, u\rangle \geq a\right\}$ and $(u=a)=\left\{x \in M_{\boldsymbol{R}}\right.$; $\langle x, u\rangle=a\}$. Each element $x$ of $P$ is contained in the relative interior of a unique face of $P$, and the cone $(P-x)^{\vee}$ is determined by the face. By this correspondence, $\operatorname{Fan}(P)$ is in bijective correspondence with the set of faces of $P$. If $\sigma \in \operatorname{Fan}(P)$ corresponds to a face $Q$ of $P$, then we have the equality $\operatorname{dim} \sigma+\operatorname{dim} Q=r$. If another cone $\tau \in \operatorname{Fan}(P)$ corresponds to a face $R$, then $R \subset Q$ if and only if $\sigma \prec \tau$. It follows that $\sigma$ and $\tau$ are faces of a common $\rho \in \operatorname{Fan}(P)$ if and only if $Q \cap R \neq \emptyset$.

We define $\operatorname{Fan}(P)$ similarly for $P$ of dimension less than $r$. In this case, $\operatorname{Fan}(P)$ consists of cones which are not strongly convex. The support of $\operatorname{Fan}(P)$ is also $C$.

In case $C=N_{\boldsymbol{R}}, P$ is a convex polytope. If $\operatorname{dim} P=r$, then $\operatorname{Fan}(P)$ is a complete fan. We call Fan $(P)$ the projective (real) fan defined by $P$ (cf. [OP, p. 383, Remark]). It is common to call it a polytopal fan, but we adopt this terminology instead for the convenience to translate Nagata's proof. If $P$ is rational, then $\operatorname{Fan}(P)$ defines a projective toric variety. 
Let $\pi$ be a strongly convex rational polyhedral cone. Then $M \cap \pi^{\vee}$ is a finitely generated semigroup with the unit 0 . If a subset $S$ of $m_{0}+M \cap \pi^{\vee}$ for some $m_{0} \in M$ satisfies the "semigroup ideal" condition

$$
m \in S, m^{\prime} \in M \cap \pi^{\vee} \Rightarrow m+m^{\prime} \in S,
$$

then there exist a finite number elements $m_{1}, \ldots, m_{s} \in S$ with

$$
S=\bigcup_{i=1}^{s}\left(m_{i}+M \cap \pi^{\vee}\right) .
$$

This fact is checked as follows. For an arbitrary field $k$, we consider the semigroup ring

$$
k\left[M \cap \pi^{\vee}\right]:=\bigoplus_{m \in M \cap \pi^{\vee}} k e(m) .
$$

Then the vector subspace $I$ generated by $\left\{\boldsymbol{e}\left(m-m_{0}\right) ; m \in S\right\}$ is an ideal. Since $k\left[M \cap \pi^{\vee}\right]$ is Noetherian, we can find a finite set of generators $\left\{\boldsymbol{e}\left(m_{1}-m_{0}\right), \ldots, \boldsymbol{e}\left(m_{s}-m_{0}\right)\right\}$ of the ideal. Then $m_{1}, \ldots, m_{s}$ satisfies the condition.

The convex hull of the above $S$ is the $\pi^{\vee}$-convex set generated by $m_{1}, \ldots, m_{s} \in S$.

3.1. The blowup of a fan at a closed subset. Let $\pi$ be a strongly convex rational polyhedral cone. For a closed proper subset $Y$ of the rational affine fan $F(\pi)$, the blowup $\mathrm{Bl}_{Y}^{M}(F(\pi))$ of $F(\pi)$ along $Y$ is defined by using the lattice $M$ as follows.

We set $\mathcal{S}(\pi):=M \cap \pi^{\vee}$, and $\mathcal{S}(\pi ; \sigma):=M \cap \pi^{\vee} \cap \sigma^{\perp}$ for each $\sigma \in F(\pi)$. The set $P(\pi, Y)$ is defined to be the convex hull of

$$
S=\mathcal{S}(\pi) \backslash \bigcup_{\sigma \in Y} \mathcal{S}(\pi ; \sigma) .
$$

$S$ is nonempty, since the zero cone $\mathbf{0}=\{0\}$ is not in $Y$, and $P(\pi, Y)$ is a $\pi^{\vee}$-convex polyhedron, since $S$ satisfies (4). Then $\operatorname{Bl}_{Y}^{M}(F(\pi)):=\operatorname{Fan}(P(\pi, Y))$ is a finite fan with support $\pi$.

The morphism of toric varieties

$$
\mathrm{Bl}_{Y}^{M}(F(\pi))_{C} \rightarrow F(\pi)_{C}
$$

corresponding to this subdivision is equal to the normalization of the blowup of $F(\pi)_{C}$ along the reduced closed subvariety $Y_{C}$.

Let $X, Y$ be rational fans of $N_{\boldsymbol{R}}$. If each $\sigma \in X$ is contained in some $\rho$ in $Y$, there exists a birational morphism $X_{C} \rightarrow Y_{C}$ of the toric varieties. Then we say that the fan $X$ dominates $Y$ and write as $f: X \rightarrow Y$. This $f$ also represents the map which sends each $\sigma \in X$ to the minimal cone in $Y$ which contains $\sigma$.

For a $\pi^{\vee}$-convex polyhedron $P$ generated by a finite subset of $M_{Q}, \operatorname{Fan}(P)$ is a subdivision of $F(\pi)$, and the corresponding morphism of toric varieties is the natural morphism Proj $B \rightarrow \operatorname{Spec} C\left[M \cap \pi^{\vee}\right]$ defined for the graded ring

$$
B:=\bigoplus_{n=0}^{\infty}[M \cap n P]_{C},
$$


where, for a subset $F$ of $M$, we denote by $F_{\boldsymbol{C}}$ the vector subspace $\bigoplus_{m \in F} \boldsymbol{C e}(m)$ of $\boldsymbol{C}[M]$. We understand $0 P=\pi^{\vee}$.

3.2. General blowups of fans. Let $X$ be a fan. We consider a set $I=\left\{I_{\sigma} ; \sigma \in X\right\}$ of subsets of $M_{\boldsymbol{R}}$ such that each $I_{\sigma}$ is $\sigma^{\vee}$-convex and the equality $I_{\sigma}=I_{\tau}+\sigma^{\vee}$ holds for any $\sigma, \tau \in X$ with the relation $\sigma \prec \tau$. Then

$$
\operatorname{Fan}_{X}(I):=\bigcup_{\sigma \in X} \operatorname{Fan}\left(I_{\sigma}\right)
$$

is a subdivision of the fan $X$. If everything is rational, this subdivision corresponds to the normalization of the blowup of a toric variety along a fractional ideal. Hence we use similar terminology for fans. Namely, we call $I=\left\{I_{\sigma} ; \sigma \in X\right\}$ a polyhedral fractional ideal of $X$, and $\operatorname{Fan}_{X}(I)$ the blowup of $X$ along $I$. We call $I$ a polyhedral ideal if $I_{\sigma} \subset \sigma^{\vee}$ for every $\sigma \in X$. For a polyhedral ideal $I$, we define the support of $I$ by $\left\{\sigma \in X ; I_{\sigma} \neq \sigma^{\vee}\right\}$. We say that $I$ is unitary at $\sigma$ if $I_{\sigma}=\sigma^{\vee}$. Namely, $I$ is unitary on the open subset $X \backslash Y$ if $Y$ is the support of $I$.

3.3. The composite of blowups.

THEOREM 3.1. Let $X$ be a finite fan and $I=\left\{I_{\sigma} ; \sigma \in X\right\}$ a polyhedral fractional ideal. We set $X^{\prime}:=\operatorname{Fan}_{X}(I)$. Let $I^{\prime}=\left\{I_{\rho}^{\prime} ; \rho \in X^{\prime}\right\}$ be a polyhedral fractional ideal of $X^{\prime}$. For a positive real number $a$, we define an ideal $J=\left\{J_{\sigma} ; \sigma \in X\right\}$ of $X$ by

$$
J_{\sigma}=\bigcap_{\substack{\rho \in X^{\prime} \\ \rho \subset \sigma}}\left(a I_{\sigma}+I_{\rho}^{\prime}\right)
$$

for $\sigma \in X$. Then, there exists a positive real number $a_{0}$ such that we have the equality $\operatorname{Fan}_{X}(J)=\operatorname{Fan}_{X^{\prime}}\left(I^{\prime}\right)$ for any $a \geq a_{0}$. In particular, the fan $\operatorname{Fan}_{X}(J)$ does not depend on the choice of $a \geq a_{0}$. If $I$ and $I^{\prime}$ are polyhedral ideals, then so is $J$.

First, we prove the following lemma.

Lemma 3.2. Let $C$ be a polyhedral cone of $N_{\boldsymbol{R}}$, and $P \subset M_{\boldsymbol{R}}$ an $r$-dimensional $C^{\vee}$ convex polyhedron. We denote by $X_{P}:=\operatorname{Fan}(P)$ the fan defined by $P$. Let $K=\left(K_{\sigma}\right)$ be a polyhedral fractional ideal of $X_{P}$. We define $a C^{\vee}$-convex set $Q$ by

$$
Q=\bigcap_{\sigma \in X_{P}}\left(a P+K_{\sigma}\right)
$$

for a positive real number $a$. Then there exists a positive real number $a_{1}$ such that the fan $X_{Q}:=\operatorname{Fan}(Q)$ is equal to $\operatorname{Fan}_{X_{P}}(K)$ for any $a \geq a_{1}$. In particular, the fan $X_{Q}$ does not depend on the choice of $a \geq a_{1}$.

PROOF. For each $\sigma \in X_{P}$, we take an element $y_{\sigma} \in P$ with $\sigma=\left(P-y_{\sigma}\right)^{\vee}$. Since $a\left(P-y_{\sigma}\right) \subset \sigma^{\vee}$ and $K_{\sigma}=K_{\sigma}+\sigma^{\vee}$, we have

$$
a P+K_{\sigma}=a y_{\sigma}+a\left(P-y_{\sigma}\right)+K_{\sigma}=a y_{\sigma}+K_{\sigma}
$$

for each $\sigma$. This implies that the support of the fan $\operatorname{Fan}\left(a P+K_{\sigma}\right)$ is $\sigma$, and the support of the fan $X_{Q}$ defined by the intersection $Q$ of these convex sets is $C$, i.e., the support of the fan $X_{P}$. 
The support of $\operatorname{Fan}_{X_{P}}(K)$ is also $C$, since it is a subdivision of $X_{P}$. Hence, for the equality $X_{Q}=\operatorname{Fan}_{X_{P}}(K)$, it suffices to show that every $\eta \in \operatorname{Fan}_{X_{P}}(K)$ is a member of $X_{Q}$.

For each $\eta$, there exist $\sigma \in X_{P}$ and $z \in K_{\sigma}$ with $\eta=\left(K_{\sigma}-z\right)^{\vee}$. Let $\tau$ be an arbitrary element of $X_{P}$. Then the cones $\sigma$ and $\tau$ are separated by $y_{\tau}-y_{\sigma}$. Actually, $\sigma \subset\left(y_{\tau}-y_{\sigma} \geq 0\right)$, since $y_{\tau}-y_{\sigma} \in P-y_{\sigma}$, while $\tau \subset\left(y_{\tau}-y_{\sigma} \leq 0\right)$, since $y_{\sigma}-y_{\tau} \in P-y_{\tau}$. We also have the equality $\sigma \cap\left(y_{\tau}-y_{\sigma}=0\right)=\tau \cap\left(y_{\tau}-y_{\sigma}=0\right)$, since the restrictions of $\left(P-y_{\sigma}\right)^{\vee}$ and $\left(P-y_{\tau}\right)^{\vee}$ to $\left(y_{\tau}-y_{\sigma}=0\right)$ are equal. Hence $\boldsymbol{R}_{0}\left(y_{\tau}-y_{\sigma}\right)+K_{\tau}=K_{\rho}$ for $\rho=\tau \cap \sigma$. Since $K_{\sigma} \subset K_{\rho}$, the convex set $a\left(y_{\tau}-y_{\sigma}\right)+K_{\tau}$ contains a neighborhood $U$ of $z$ in $K_{\sigma}$ for sufficiently large $a$. Then $a y_{\sigma}+z$ is an element of $a y_{\tau}+K_{\tau}$, and

$$
\left(\left(a y_{\tau}+K_{\tau}\right)-\left(a y_{\sigma}+z\right)\right)^{\vee} \subset(U-z)^{\vee}=\left(K_{\sigma}-z\right)^{\vee}=\eta .
$$

If we take the real number $a$ sufficiently large for all $\tau \in X_{P}$, then $a y_{\sigma}+z$ is in $Q$. Since $\left(Q-\left(a y_{\sigma}+z\right)\right)^{\vee}$ is equal to the sum of the first terms of (6) for all $\tau \in X_{P}$, it is equal to $\eta$ for such $a$. Hence $\eta$ is in $X_{Q}$.

Since $\operatorname{Fan}_{X_{P}}(K)$ is a finite fan, every $\eta \in \operatorname{Fan}_{X_{P}}(K)$ is in $X_{Q}$ for sufficiently large $a \geq 0$.

Proof of Theorem 3.1. $\operatorname{Fan}_{X}(J)$ and $\operatorname{Fan}_{X^{\prime}}\left(I^{\prime}\right)$ are subdivisions of $X$ by definitions. We apply Lemma 3.2 to $P=I_{\sigma}$ and $K$, which is defined to be the restriction of $J$ to $\left\{\rho \in X^{\prime} ; \rho \subset \sigma\right\}$. Then we know that $\operatorname{Fan}_{X}(J)$ and $\operatorname{Fan}_{X^{\prime}}\left(I^{\prime}\right)$ are equal on the cone $\sigma$ for sufficiently large $a \geq 0$. Since $X$ is finite, there exists $a_{0} \geq 0$ such that $\operatorname{Fan}_{X}(J)=\operatorname{Fan}_{X^{\prime}}\left(I^{\prime}\right)$ for every $a \geq a_{0}$.

The last assertion of the theorem is clear from the first part.

3.4. Sums and intersections of ideals. Let $I=\left\{I_{\sigma} ; \sigma \in X\right\}$ and $J=\left\{J_{\sigma} ; \sigma \in X\right\}$ be polyhedral fractional ideals of $X$. The sum $I+J$ of these ideals is defined to be $\left\{I_{\sigma}+J_{\sigma}\right.$; $\sigma \in X\}$. This is an analog of the product of fractional ideals of an integral scheme. Since $I_{\sigma} \cap J_{\sigma}$ is a $\sigma^{\vee}$-convex polyhedron, $I \cap J:=\left\{I_{\sigma} \cap J_{\sigma} ; \sigma \in X\right\}$ is also a fractional ideal of $X$.

For finite fans $X, X^{\prime}$, we define the join by

$$
J\left(X, X^{\prime}\right):=\left\{\sigma \cap \tau ; \sigma \in X, \tau \in X^{\prime}\right\} .
$$

Then $J\left(X, X^{\prime}\right)$ is also a finite fan, and dominates both $X$ and $X^{\prime}$. If a fan $Y$ dominates both $X$ and $X^{\prime}$, then the join $J\left(X, X^{\prime}\right)$ is also dominated by $Y$. The equality

$$
\mathrm{ZR}\left(J\left(X, X^{\prime}\right)\right)=\mathrm{ZR}(X) \cap \mathrm{ZR}\left(X^{\prime}\right)
$$

is checked easily.

PROPOSITION 3.3. For polyhedral fractional ideals $I, J$ of $X$, we have the equality

$$
\operatorname{Fan}_{X}(I+J)=J\left(\operatorname{Fan}_{X}(I), \operatorname{Fan}_{X}(J)\right) .
$$

Proof. Since both fans are subdivisions of $X$, it suffices to show that they define the same subdivision on each $\sigma \in X$. We set $P=I_{\sigma}$ and $Q=J_{\sigma}$. For $\rho \in \operatorname{Fan}_{X}(I+J)$ 
contained in $\sigma$, there exists $z \in P+Q$ with $\rho=(P+Q-z)^{\vee}$. For $x \in P$ and $y \in Q$ with $z=x+y$, we have the equalities

$$
(P+Q-z)^{\vee}=((P-x)+(Q-y))^{\vee}=(P-x)^{\vee} \cap(Q-y)^{\vee} .
$$

Hence $\rho \in J\left(\operatorname{Fan}_{X}(I), \operatorname{Fan}_{X}(J)\right)$. By the same equalities, we know that each element of $J\left(\operatorname{Fan}_{X}(I), \operatorname{Fan}_{X}(J)\right)$ is a member of $\operatorname{Fan}_{X}(I+J)$.

For a polyhedral fractional ideal $I$, the polyhedral fractional ideal $I^{-1}$ is defined by

$$
I_{\sigma}^{-1}:=\left\{x \in M_{\boldsymbol{R}} ; I_{\sigma}+x \subset \sigma^{\vee}\right\} .
$$

Then $I+I^{-1}$ is a polyhedral ideal of $X$, since $I_{\sigma}+I_{\sigma}^{-1} \subset \sigma^{\vee}$ for every $\sigma$. The fan $\operatorname{Fan}_{X}\left(I+I^{-1}\right)$ is a subdivision of $\operatorname{Fan}_{X}(I)$ by Proposition 3.3.

3.5. The maximal extension of an ideal. Let $X$ be a fan, and $U$ a subfan of $X$. For a polyhedral ideal $I$ of $U$, there exists the largest polyhedral ideal $I^{\prime}$ of $X$ with $I^{\prime} \mid U=I$. It is obtained by setting

$$
I_{\sigma}^{\prime}:=\sigma^{\vee} \cap \bigcap_{\eta \in F(\sigma) \cap U} I_{\eta}
$$

for each $\sigma \in X$.

3.6. The primary decomposition. Let $\sigma$ be an element of a fan $X$. A polyhedral ideal $I$ of the affine fan $F(\sigma)$ is said to be primary if $\sigma^{\vee} \backslash I_{\sigma}$ is nonempty and $\sigma^{\perp}$-bounded, where we say that a subset $S \subset M_{R}$ is $\sigma^{\perp}$-bounded if $S=S+\sigma^{\perp}$ and if the image of $S$ in $M_{\boldsymbol{R}} / \sigma^{\perp}$ is bounded. In this case, the equality $I_{\eta}=\eta^{\vee}$ holds for any $\eta \in F(\sigma) \backslash\{\sigma\}$. Conversely, $I$ is primary if it satisfies this condition. The ideal $I$ is primary if and only if, for any $m \in M \cap\left(\sigma^{\vee} \backslash \sigma^{\perp}\right)$, there exists a positive integer $c$ with $\mathrm{cm} \in I_{\sigma}$. The maximal extension of a primary polyhedral ideal $I$ to $X$ is called the primary polyhedral ideal of $X$ at $\sigma$. If we denote also by $I$ the the extended ideal, $I_{\rho}$ is unitary unless $\sigma \prec \rho$. Namely, the support of the primary ideal is contained in the closure of $\{\sigma\}$ in $X$.

PROPOSITION 3.4. Let I be a polyhedral ideal of $X$ with support $Y$. Then there exists a set $\left\{I^{\sigma} ; \sigma \in X\right\}$ of polyhedral ideals of $X$, such that each $I^{\sigma}$ is unitary on $X$ or primary at $\sigma$ and

$$
I=\bigcap_{\sigma \in X} I^{\sigma}=\bigcap_{\sigma \in Y} I^{\sigma} .
$$

Here the right-hand side is essentially a finite intersection for each $\rho \in X$ even if $X$ is not finite.

Proof. Proposition 3.4 is equivalent to the assertion that there exists $\left\{P_{\sigma} ; \sigma \in X\right\}$ such that $\sigma^{\vee} \backslash P_{\sigma}$ is $\sigma^{\perp}$-bounded for every $\sigma$ and

$$
I_{\rho}=\bigcap_{\sigma \in F(\rho)} P_{\sigma}
$$


for every $\rho \in X$. The construction of $P_{\sigma}$ is done inductively from low dimensional cones. We set $P_{\sigma}=\sigma^{\vee}$ for $\sigma$ outside $Y$. Assume that $P_{\eta}$ is determined for every $\eta$ in $F(\sigma) \backslash\{\sigma\}$. Then

$$
I_{\sigma} \backslash \bigcap_{\eta \in F(\sigma) \backslash\{\sigma\}} P_{\eta}
$$

is $\sigma^{\perp}$-bounded. Let $Q_{1}, \ldots, Q_{s}$ be the $\sigma^{\perp}$-bounded faces of codimension one of $I_{\sigma}$. We take $y_{1}, \ldots, y_{s} \in N_{\boldsymbol{R}}$ and $c_{1}, \ldots, c_{s} \in \boldsymbol{R}$ with

$$
I_{\sigma} \subset\left(y_{i} \geq c_{i}\right), \quad Q_{i}=I_{\sigma} \cap\left(y_{i}=c_{i}\right), \quad i=1, \ldots, s .
$$

Then we have $y_{1}, \ldots, y_{s} \in$ rel. int $\sigma$. Hence

$$
P_{\sigma}:=\sigma^{\vee} \cap \bigcap_{i=1}^{s}\left(y_{i} \geq c_{i}\right)
$$

satisfies the condition. Since $I^{\sigma}$ is trivial for $\sigma \in X \backslash Y$, we get the last equality of (7).

The last assertion follows from the fact that $I_{\rho}^{\sigma}=\rho^{\vee}$ for $\sigma \notin F(\rho)$,

3.7. Local blowups. Let $I$ be a polyhedral ideal of a fan $X$, and $U$ an open subset of $X$. For the support $Y$ of $I$, we have a primary decomposition

$$
I=\bigcap_{\sigma \in Y} I^{\sigma}
$$

by Proposition 3.4. If we set

$$
I^{\prime}=\bigcap_{\sigma \in Y \cap U} I^{\sigma},
$$

then $I^{\prime}$ and $I$ are equal on $U$. On the other hand, if $\rho \in X$ is not contained in the closure of $Y \cap U$, then $I_{\rho}^{\prime}=\rho^{\vee}$. Hence the $\operatorname{blowup} \operatorname{Fan}_{X}\left(I^{\prime}\right)$ is equal to $\operatorname{Fan}_{X}(I)$ on $U$ and to $X$ on $X \backslash \overline{Y \cap U}$. This localization of the blowup is not possible in general for a polyhedral fractional ideal.

3.8. Some lemmas. Let $Q \subset M_{\boldsymbol{R}}$ be a rational convex polytope, i.e., a convex closure of a finite set of rational points. Then, for any polyhedral cone $\sigma, I(Q)_{\sigma}:=Q+\sigma^{\vee}$ is a $\sigma^{\vee}$-convex subset.

Let $X$ be a fan. Then $I(Q, X):=\left\{I(Q)_{\sigma} ; \sigma \in X\right\}$ is a polyhedral fractional ideal. Hence $\operatorname{Fan}_{X}(I(Q, X))$ is a subdivision of $X$. If $Q$ is $r$-dimensional, then $\operatorname{Fan}(Q)$ is projective and $\operatorname{Fan}_{X}(I(Q, X))$ is the join $J(\operatorname{Fan}(Q), X)$ of $\operatorname{Fan}(Q)$ and $X . \operatorname{In} \operatorname{particular}, \operatorname{Fan}_{X}(I(Q, X))$ dominates the projective fan $\operatorname{Fan}(Q)$.

For the polyhedral ideal $I(Q, X)+I(Q, X)^{-1}$ of $X$, the fan $\operatorname{Fan}_{X}\left(I(Q, X)+I(Q, X)^{-1}\right)$ is a subdivision of $\operatorname{Fan}_{X}(I(Q, X))$. In particular, it dominates both $X$ and $\operatorname{Fan}(Q)$. Since $\left(I(Q, X)+I(Q, X)^{-1}\right)_{\gamma}=\gamma^{\vee}$ for $\gamma$ with $\operatorname{dim} \gamma \leq 1$, the support of this ideal consists of cones of dimension at least two. If $\sigma$ is contained in both $X$ and $\operatorname{Fan}(P)$, then the polyhedral ideals $I(Q, X)$ and $I(Q, X)^{-1}$ are unitary at $\sigma$. Hence the blowup

$$
\operatorname{Fan}_{X}\left(I(Q, X)+I(Q, X)^{-1}\right) \rightarrow X
$$

does not subdivide the cone $\sigma$. Since this is a blowup along a polyhedral ideal, local blowups are possible for any subfan of $X$. 
LEMMA 3.5. Suppose that $v \in \mathrm{ZR}(M)$ dominates $\tau$ and the first generalization $w$ of $v$ dominates $\sigma$. We define

$$
P:=\operatorname{conv}\left\{m \in M \cap \tau^{\vee} ; m_{0} \leq v m\right\}
$$

for an element $m_{0} \in M \cap\left(\tau^{\vee} \cap \sigma^{\perp}\right)$. Then $P$ defines a primary polyhedral ideal of $F(\tau)$.

Proof. Clearly, $P$ is a $\tau^{\vee}$-convex subset of $\tau^{\vee}$. It suffices to show that, for any element $m \in M \cap\left(\tau^{\vee} \backslash \tau^{\perp}\right), c m$ is in $P$ for a sufficiently large integer $c$.

If $m \in M \cap\left(\tau^{\vee} \backslash \sigma^{\perp}\right)$, then $m_{0}<_{w} m$, since $m_{0} \in M \cap \sigma^{\perp}$. Hence $m_{0} \leq_{v} m$ and $m \in P$.

Suppose $m \in M \cap \tau^{\vee} \cap\left(\sigma^{\perp} \backslash \tau^{\perp}\right)$. Since $v$ dominates $\tau, M \cap \tau^{\vee} \backslash \tau^{\perp}$ is a subset of $L(v) \backslash L^{0}(v)$. Hence $0<v m$. Let $s$ be the rank of $v$, and $\left(y_{0}, \ldots, y_{s-1}\right)$ a defining sequence of it. Then linear functions $y_{0}, \ldots, y_{s-2}$ are zero on $M \cap \sigma^{\perp}$, and

$$
x_{1} \leq_{v} x_{2} \Leftrightarrow\left\langle x_{1}, y_{s-1}\right\rangle \leq\left\langle x_{2}, y_{s-1}\right\rangle
$$

for $x_{1}, x_{2} \in M \cap \sigma^{\perp}$. We have $\left\langle m, y_{s-1}\right\rangle>0$, since $m \in M \cap \sigma^{\perp}$ and $0<_{v} m$. Hence we have $\left\langle m_{0}, y_{s-1}\right\rangle \leq\left\langle\mathrm{cm}, y_{s-1}\right\rangle$ for sufficiently large $c$. Then $m_{0} \leq_{v} \mathrm{~cm}$ and $\mathrm{cm} \in P$.

LEMMA 3.6. Let $X$ be a fan and $U$ an open subset of it. Let $Y_{1}, Y_{2}$ be closed subsets of $U$ with $Y_{1} \cap Y_{2}=\emptyset$. For a polyhedral ideal $I$ of $X$ with the support $Y:=\bar{Y}_{1} \cap \bar{Y}_{2}$, let $X^{\prime}$ be the blowup of $X$ at $I$. Then, if we regard $U$ an open subset of $X^{\prime}$, then the closures of $Y_{1}$ and $Y_{2}$ in $X^{\prime}$ are disjoint.

Proof. Since $Y$ does not intersect $U$, this blowup leaves $U$ unchanged. It suffices to show that $\sigma \in Y_{1}$ and $\tau \in Y_{2}$ cannot be faces of a common element of $X^{\prime}$. Since $X$ is covered by affine fans, we may assume that $X=F(\pi)$ and that $\sigma$ and $\tau$ are faces of $\pi$. Since $\sigma$ and $\tau$ are outside $Y, I_{\pi} \cap \sigma^{\perp}$ and $I_{\pi} \cap \tau^{\perp}$ are nonempty. Let $\rho \in F(\pi)$ be the minimal face of $\pi$ which contains $\sigma$ and $\tau$. Then $I_{\pi}$ does not intersect $\pi^{\vee} \cap \rho^{\perp}$, since $\rho \in Y$. Since the intersection of $\pi^{\vee} \cap \sigma^{\perp}$ and $\pi^{\vee} \cap \tau^{\perp}$ is $\pi^{\vee} \cap \rho^{\perp}$, we have

$$
\left(I_{\pi} \cap \sigma^{\perp}\right) \cap\left(I_{\pi} \cap \tau^{\perp}\right)=\emptyset .
$$

Hence there is no cone in $X^{\prime}=\operatorname{Fan}\left(I_{\pi}\right)$ which contains both $\sigma$ and $\tau$.

4. Completions of fans. In this section, we assume that fans are rational, and we prove the following theorem. A similar theorem for not necessarily rational fans will be proved in the next section as the second proof of Theorem 1.1.

THEOREM 4.1. Let $X$ be a finite (rational) fan. Then there exists a complete fan $X^{\prime}$ such that $X$ is a subfan of $X^{\prime}$.

Any affine fan has a completion by the following lemma.

LEMMA 4.2. For a rational polyhedral cone $\sigma$, there exists a projective fan $X$ which contains $\sigma$ as an element. 
Proof. First, we consider the case $\sigma=\mathbf{0}$. Let $\left\{m_{1}, \ldots, m_{r}\right\}$ and $\left\{n_{1}, \ldots, n_{r}\right\}$ be mutually dual basis of $M$ and $N$. We set $n_{0}:=-\left(n_{1}+\cdots+n_{r}\right)$. The fan $\Pi_{r}$ is defined to be the set of cones generated by proper subsets of $\left\{n_{0}, n_{1}, \ldots, n_{r}\right\}$. This is equal to the fan $\operatorname{Fan}(P)$ for the convex closure $P$ of $\left\{0, m_{1}, \ldots, m_{r}\right\}$. This is a complete fan and of course contains $\mathbf{0}$. The associated toric variety of this fan is the $r$-dimensional projective space (cf. [O1, p. 96]).

In case $\operatorname{dim} \sigma=r$, we take $n \in N \cap \operatorname{int} \sigma$ and set

$$
Y:=F(\sigma) \cup\left\{\eta+\boldsymbol{R}_{0}(-n) ; \eta \in F(\sigma) \backslash\{\sigma\}\right\} .
$$

Then $Y$ is equal to the projective fan $\operatorname{Fan}(P)$ for the $r$-dimensional convex polytope $P=\{x \in$ $\left.\sigma^{\vee} ;\langle x, n\rangle \leq 1\right\}$, and $\sigma \in Y$.

In the general case, let $s=\operatorname{dim} \sigma$. We take a decomposition $N=N^{\prime} \oplus N^{\prime \prime}$ such that $\sigma$ is a maximal dimensional cone in $N_{\boldsymbol{R}}^{\prime}$. Then the product fan of a complete fan of $N_{\boldsymbol{R}}^{\prime}$ which contains $\sigma$ and $\Pi_{r-s}$ of $N_{\boldsymbol{R}}^{\prime \prime}$ for a basis satisfies the condition.

THEOREM 4.3. The following conditions on a fan $X$ are equivalent.

(1) $X$ is complete, i.e., $X$ is finite and $|X|=N_{\boldsymbol{R}}$.

(2) The equality $\mathrm{ZR}(X)=\mathrm{ZR}(M)$ holds.

(3) The equality $\bigcup_{\sigma \in X} \operatorname{dom} \sigma=\mathrm{ZR}(M)$ holds.

Proof. (2) and (3) are equivalent since $\mathrm{ZR}(X)=\bigcup_{\sigma \in X} \operatorname{dom} \sigma$ by Lemma 2.9.

Suppose (2) holds. Since $\{\|\sigma\| ; \sigma \in X\}$ is an open covering of $\operatorname{ZR}(M)$, there exists a finite subfan $X^{\prime} \subset X$ with $\bigcup_{\sigma \in X^{\prime}}\|\sigma\|=\mathrm{ZR}(M)$ by Theorem 2.5. Since $\operatorname{dom}(\sigma)$ 's are nonempty and disjoint for $\sigma \in X$, we have $X^{\prime}=X$. Hence $X$ is finite. Take an arbitrary element $y \in N_{\boldsymbol{R}}$ and consider $v \in \mathrm{ZR}(M)^{1}$ with $L(v)=M \cap(y \geq 0)$. By assumption and Lemma 2.9, there exists a cone $\sigma \in X$ with $M \cap \sigma^{\vee} \subset L(v) \subset(y \geq 0)$. Then $y \in \sigma \subset|X|$. Hence $|X|=N_{\boldsymbol{R}}$ and $X$ is complete.

Suppose (1) holds and take an arbitrary element $v \in \mathrm{ZR}(M)$. Let $s$ be the rank of $v$ and $\left(y_{0}, \ldots, y_{s-1}\right)$ a defining sequence of $v$. Since $|X|=N_{\boldsymbol{R}}, z_{\varepsilon}:=\sum_{i=0}^{s-1} \varepsilon^{i} y_{i}$ is contained in the relative interior of a cone of $X$ for every $\varepsilon>0$. Since $X$ is a finite fan, there exist $\tau \in X$ and a sequence $\left\{\varepsilon_{j}\right\}$ of positive real numbers with the limit 0 such that all $z_{\varepsilon_{j}}$ 's are contained in rel. int $\tau$. Then $v \in \operatorname{dom} \tau$ by Lemma 2.6. This implies (3).

For finite fans $X, X^{\prime}$, we denote by $X \cap X^{\prime}$ the set of cones contained in both $X$ and $X^{\prime}$. Clearly, $X \cap X^{\prime}$ is a subfan of both $X$ and $X^{\prime}$. We set

$$
D_{X, X^{\prime}}:=\left\{\sigma \in X \text {; there exists } \tau \in X^{\prime} \text { such that } \sigma \subset \tau\right\} \text {. }
$$

This is the maximal subfan of $X$ which dominates $X^{\prime}$. Clearly, we have

$$
D_{X, X^{\prime}} \cap D_{X^{\prime}, X}=X \cap X^{\prime} .
$$

The fan

$$
J\left(X, X^{\prime}\right):=\left\{\sigma \cap \tau ; \sigma \in X, \tau \in X^{\prime}\right\}
$$

defined in Section 3 is finite and dominates $X$ and $X^{\prime}$. 
We say $X^{\prime}$ to be quasi-dominant over $X$ if $J\left(X, X^{\prime}\right)$ is a subfan of $X^{\prime}$. Then $J\left(X, X^{\prime}\right)=$ $D_{X^{\prime}, X}$. If $\mathrm{ZR}(X) \subset \mathrm{ZR}\left(X^{\prime}\right)$ and $X^{\prime}$ is quasi-dominant over $X$, then $J\left(X, X^{\prime}\right)$ is a subfan of $X^{\prime}$ and is a subdivision of $X$. In particular, if a complete fan $X^{*}$ is quasi-dominant over $X$, then a subfan of $X^{*}$ is a subdivision of $X$.

More generally, we define the join $J\left(X_{1}, \ldots, X_{S}\right)$ for a finite number of finite fans $X_{1}, \ldots, X_{s}(s \geq 1)$. Namely, we inductively define $J\left(X_{1}\right):=X_{1}$ and

$$
J\left(X_{1}, \ldots, X_{i}\right)=J\left(J\left(X_{1}, \ldots, X_{i-1}\right), X_{i}\right)
$$

for $i=2, \ldots, s$. It is easy to see that $J\left(X_{1}, \ldots, X_{s}\right)$ is independent of the order of $X_{1}, \ldots, X_{s}$.

As we mentioned in Section $3, J(X, Y)$ is equal to $\operatorname{Fan}(P+Q)$ if $X=\operatorname{Fan}(P)$ and $Y=\operatorname{Fan}(Q)$ for convex polyhedra $P$ and $Q$.

The following theorem is "Chow's lemma" for a fan.

THEOREM 4.4. For a finite fan $X$, there exists a projective fan $X^{*}$ such that a subfan $X^{\prime}$ of $X^{*}$ is a subdivision of $X$, i.e., $X^{*}$ is quasi-dominant over $X$.

Proof. Let $\left\{\sigma_{1}, \ldots, \sigma_{s}\right\}$ be the set of maximal elements of $X$. By Lemma 4.2, there exists a projective fan $X_{i}$ which contains $\sigma_{i}$ as an element for each $i$. Then $X^{*}:=J\left(X_{1}, \ldots, X_{s}\right)$ is a projective fan. Clearly, each $\sigma_{i}$ is a union of cones in $X^{*}$. Hence the set $X^{\prime} \subset X^{*}$ of cones contained in one of $\sigma_{i}$ 's is a subdivision of $X$.

THEOREM 4.5. Let $X_{1}, X_{2}$ be finite fans and $v$ an element of $\operatorname{ZR}\left(X_{1}\right) \cap \operatorname{ZR}\left(X_{2}\right)$. Then there exists a fan $X_{v}$ with the following properties: (1) $X_{v}$ is the blowup at a polyhedral ideal I of $X_{1}$. (2) The ideal I of (1) is unitary at $D_{X_{1}, X_{2}}$ and hence $D_{X_{1}, X_{2}} \subset X_{v}$. (3) If $v$ dominates $\tau_{v} \in X_{v}$ and $\tau_{2} \in X_{2}$, then $\tau_{v} \subset \tau_{2}$.

PROOF. We prove the theorem by induction on the rank of $v$. If $v=\eta(M)$, then $v$ dominates 0 and $X_{v}:=X_{1}$ satisfies the condition.

Assume that the rank is at least one. Let $\tau_{1} \in X_{1}$ and $\tau_{2} \in X_{2}$ be the cones dominated by $v$. If $\tau_{1} \subset \tau_{2}$, then $X_{v}:=X_{1}$ is enough. Hence we assume that $\tau_{1}$ is not contained in $\tau_{2}$. In particular, $\tau_{1} \notin D_{X_{1}, X_{2}}$.

Let $w$ be the first generalization of $v$. By Lemma 2.10, $w$ dominates a face $\sigma_{1}$ of $\tau_{1}$ and a face $\sigma_{2}$ of $\tau_{2}$. Since $\operatorname{rank}(w)=\operatorname{rank}(v)-1$, we can apply the induction assumption for $w$. Hence, by replacing $X_{1}$ by $X_{w}$, we may assume $\sigma_{1} \subset \sigma_{2}$. Here, recall that the composite of blowups is a blowup by Theorem 3.1. Assume that the semigroup $M \cap \tau_{2}^{\vee}$ is generated by $m_{1}, \ldots, m_{s}$. Since $\sigma_{1} \subset \sigma_{2} \subset \tau_{2}$, we have

$$
M \cap \tau_{2}^{\vee} \subset M \cap \sigma_{2}^{\vee} \subset M \cap \sigma_{1}^{\vee} .
$$

Hence, there exists $m_{0} \in M \cap \operatorname{rel} \operatorname{int}\left(\tau_{1}^{\vee} \cap \sigma_{1}^{\perp}\right)$ with

$$
m_{1}, \ldots, m_{s} \in-m_{0}+M \cap \tau_{1}^{\vee}
$$

[O1, Prop. 1.3].

Let $s$ be the rank of $v$ and $\left(y_{0}, \ldots, y_{s-1}\right)$ a defining sequence of $v$. Then $P:=\operatorname{conv}\{m \in$ $\left.M \cap \tau_{1}^{\vee} ; m_{0} \leq_{v} m\right\}$ defines a primary polyhedral ideal of $F\left(\tau_{1}\right)$ by Lemma 3.5. Let $I(P)$ be 
the resulting primary polyhedral ideal of $X_{1}$, and $X_{v}$ the blowup of $X_{1}$ at $I(P)$. Since $I(P)$ is unitary at cones which do not contain $\tau_{1}$, it is unitary on $D_{X_{1}, X_{2}}$ and $D_{X_{1}, X_{2}} \subset X_{v}$ is satisfied. Since $m_{1}, \ldots, m_{s} \in L(v)$ and $m_{1}+m_{0}, \ldots, m_{s}+m_{0} \in M \cap \tau_{1}^{\vee}$, we have $m_{1}, \ldots, m_{s} \in$ $\boldsymbol{R}\left(P-m_{0}\right)$. The cone $\boldsymbol{R}\left(P-m_{0}\right)^{\vee}$ of $X_{v}$ is contained in the cone $\tau_{2}$, since $\left\{m_{1}, \ldots, m_{s}\right\}$ generates $\tau_{2}^{\vee}$. Since $M \cap \boldsymbol{R}\left(P-m_{0}\right) \subset L(v), v$ dominates a face $\tau_{v}$ of $\boldsymbol{R}\left(P-m_{0}\right)^{\vee}$ by Lemma 2.9. Clearly, $\tau_{v} \in X_{v}$ and $\tau_{v} \subset \tau_{2}$.

THEOREM 4.6. Let $X, X^{\prime}$ be finite fans. Then there exists a fan $X^{*}$ with the following properties: (1) $X^{*}$ is a blowup of $X$ along a polyhedral ideal I. (2) I is unitary on $D_{X, X^{\prime}}$ and $D_{X, X^{\prime}} \subset X^{*}$. (3) $X^{*}$ is quasi-dominant over $X^{\prime}$.

Proof. For each element $v \in \mathrm{ZR}\left(J\left(X, X^{\prime}\right)\right)$, Theorem 4.5 says that there exists a blowup $X_{v}$ of $X$ at a polyhedral ideal which is unitary on $D_{X, X^{\prime}}$ and the cone $\sigma_{v} \in X_{v}$ dominated by $v$ is contained in some $\tau \in X^{\prime}$. Since $\left\|\sigma_{v}\right\|$ is an open neighborhood of $v$, there exist $v_{1}, \ldots, v_{s} \in \mathrm{ZR}\left(J\left(X, X^{\prime}\right)\right)$ with

$$
\operatorname{ZR}\left(J\left(X, X^{\prime}\right)\right)=\bigcup_{i=1}^{s}\left\|\sigma_{v_{i}}\right\|
$$

by the compactness of $\operatorname{ZR}\left(J\left(X, X^{\prime}\right)\right)$. We set $X^{*}:=J\left(X_{v_{1}}, \ldots, X_{v_{s}}\right)$. Then $X^{*}$ is a blowup of $X$ at a polyhedral ideal which is unitary on $D_{X, X^{\prime}}$ by Theorems 3.1 and 4.5. If $v \in$ $\operatorname{ZR}\left(J\left(X, X^{\prime}\right)\right)$ dominates $\sigma \in X^{*}$, then $\sigma$ is contained in some $\sigma_{v_{i}}$ and hence in some $\tau \in X^{\prime}$. Hence $X^{*}$ is quasi-dominant over $X^{\prime}$.

THEOREM 4.7. Let $X$ be a finite fan and $v$ an element of $\operatorname{ZR}(M)$. Then there exists a finite fan $X^{\prime}$ which contains $X$ and satisfies $v \in \mathrm{ZR}\left(X^{\prime}\right)$. Furthermore, we can take $X^{\prime}$ so that $X^{\prime} \backslash X$ is contained in a projective fan.

PROOF. We prove the first assertion of the theorem by induction on the rank of $v$.

If $v \in \mathrm{ZR}(X)$, then $X^{\prime}:=X$ is enough. Hence we assume $v \notin \mathrm{ZR}(X)$. In particular, the rank is at least one. Let $w$ be the first generalization of $v$. By the induction assumption, there exists a finite fan $X^{\prime \prime}$ which contains $X$ and contains $w$ in its Zariski-Riemann space. Hence by replacing $X$ by $X^{\prime \prime}$, we may assume $w \in \operatorname{ZR}(X)$. Then $w$ dominates a cone $\sigma \in X$.

Let $X^{*}$ be a projective fan which contains $\sigma$. By using Theorem 4.6, we replace $X^{*}$ by its blowup so that $X^{*}$ is quasi-dominant over $X$. Since $X^{*}$ is complete, a subfan $X_{1} \subset X^{*}$ is a subdivision of $X$. Let $Z^{*}:=X_{1} \backslash\left(X \cap X^{*}\right)$, and let $\overline{Z^{*}}$ be its closure in $X^{*}$. Since $X^{*}$ is complete, $v$ dominates a cone $\rho$ of $X^{*}$.

(1) First, we consider the case $\rho \notin \overline{Z^{*}}$. In this case, it suffices to show that $X^{\prime}:=$ $X \cup\left(X^{*} \backslash \overline{Z^{*}}\right)$ is a fan. Since $X^{*} \backslash \overline{Z^{*}}$ is a fan, it suffices to show that it satisfies the last condition of Proposition 2.7. Assume that $u \in \mathrm{ZR}(M)$ dominates $\tau \in X$ and $\eta \in X^{*} \backslash \overline{Z^{*}}$. Then, $\eta \in X_{1}$ since $u \in \mathrm{ZR}(X)=\mathrm{ZR}\left(X_{1}\right)$. Since $\eta \notin Z^{*} \subset \overline{Z^{*}}$, we have $\eta \in X \cap X^{*}$. Since $X$ is a fan, $\eta$ is equal to $\tau$.

(2) Now, assume $\rho \in \overline{Z^{*}} . \rho \in X^{*} \backslash X_{1}$ by the assumption $v \notin \mathrm{ZR}(X)$. Since the generalization $w$ of $v$ dominates $\sigma$, we know that $\sigma$ is a face of $\rho$. We take an element 
$m_{0} \in M \cap$ rel. $\operatorname{int}\left(\rho^{\vee} \cap \sigma^{\perp}\right)$, and set

$$
P:=\operatorname{conv}\left\{m \in M \cap \rho^{\vee} ; m_{0} \leq_{v} m\right\}
$$

Then $P$ defines a polyhedral ideal of $X^{*}$ primary at $\rho$. Let $X^{* *}$ be the blowup of $X^{*}$ along this ideal. Note that this blowup does not change any cones which do not contain $\rho$. In particular, $X$ is invariant by the blowup. Since $M \cap \boldsymbol{R}\left(P-m_{0}\right) \subset L(v)$ and $\boldsymbol{R}\left(P-m_{0}\right)^{\vee}$ is an element of $X^{* *}, v$ dominates a face $\tau$ of $\boldsymbol{R}\left(P-m_{0}\right)^{\vee}$. For the closure $\overline{Z^{* *}}$ of $Z^{* *}:=Z^{*}$ in $X^{* *}$, we will show $\tau \notin \overline{Z^{* *}}$. Suppose that a face $\eta$ of $\tau$ was contained in $Z^{*}$. Since $\eta \subset \rho$ and $\eta, \rho \in X^{*}, \eta$ is a face of $\rho$. Since $\eta \in Z^{*}$ and $\sigma \in X \cap X^{*}, \eta$ is not a face of $\sigma$. Hence $m_{0}$ is not in $\rho^{\vee} \cap \eta^{\perp}$ by [O1, Prop. A.6]. Since $\eta$ corresponds to the face $P \cap \eta^{\perp}$ of $P$ and since $m_{0}$ is not in $P \cap \eta^{\perp} \subset \rho^{\vee} \cap \eta^{\perp}, \eta$ is not a face of $\boldsymbol{R}\left(P-m_{0}\right)^{\vee}$. Hence $\eta$ is not a face of $\tau$ contrary to the assumption. Since $X \cap X^{*}=X \cap X^{* *}$, we are reduced to the case (1) by replacing $X^{*}$ by $X^{* *}$.

For the last assertion, we take $\tau \in X^{\prime}$ which is dominated by $v$. If we replace $X^{\prime}$ by $X \cup F(\tau)$, then $X^{\prime} \backslash X$ is contained in a projective fan which contains $\tau$ (cf. Lemma 4.2).

THEOREM 4.8. Let $X_{1}, X_{2}$ be finite fans, and let $X:=X_{1} \cap X_{2}$. If $X_{1} \backslash X$ is a subset of a projective fan $X^{*}$, then there exists a finite fan $X_{3}$ which contains $X$ and satisfies $\mathrm{ZR}\left(X_{3}\right)=\mathrm{ZR}\left(X_{1}\right) \cup \mathrm{ZR}\left(X_{2}\right)$.

Proof. We show later that we can replace $X_{1}, X_{2}, X^{*}$ by their subdivisions without shrinking $X=X_{1} \cap X_{2}$ so that they satisfy the following conditions:

(1) $X_{1}$ is quasi-dominant over $X_{2}$, i.e., $U_{1}=J\left(X_{1}, X_{2}\right)$ is an open subset of $X_{1}$.

(2) $U_{1}$ is a subdivision of an open subset $U_{2} \subset X_{2}$.

(3) Let $W_{1}:=U_{1} \backslash X$ and $Y:=X \backslash\left(X \cap X^{*}\right)$, and let $W_{2}$ be the image of $W_{1}$ in $U_{2}$. Then the closures $\overline{W_{2}}$ and $Y_{2}:=\bar{Y}$ in $X_{2}$ are disjoint.

(4) $X^{*}$ is quasi-dominant over $X_{2}$.

By the property (4), $U^{*}:=J\left(X_{2}, X^{*}\right)$ is an open subset of $X^{*}$, and the natural map $\psi: U^{*} \rightarrow X_{2}$ is a subdivision. (1) and (3) imply $U_{2}=X \cup W_{2}$ and $Y$ is closed in $U_{2}$.

Since $X^{*}$ is projective, there exists a blowup of $X_{2}$ of type (8) in Section 3.8. Namely, there exists a polyhedral ideal $I$ of $X_{2}$ such that $I$ is unitary on $X \cap X^{*}$ and the blowup of $X_{2}$ along $I$ is a subdivision of $U^{*}$. Let $I^{\prime}$ be the maximal extension of the restriction of the ideal $I$ to the open subset $X_{2} \backslash\left(Y_{2} \cup \overline{W_{2}}\right)$ of $X_{2}$. $I^{\prime}$ is unitary on the open set $U_{2}$, since $U_{2} \subset\left(X \cap X^{*}\right) \cup Y_{2} \cup \overline{W_{2}}$. Let $U_{2}^{*}$ be the blowup of $X_{2}$ along $I^{\prime}$, and $\phi: U_{2}^{*} \rightarrow X_{2}$ the subdivision map. Consider the two subdivision maps $U_{2}^{*} \backslash \phi^{-1}\left(Y_{2}\right) \rightarrow X_{2} \backslash Y_{2}$ and $U^{*} \backslash \psi^{-1}\left(Y_{2}\right) \rightarrow X_{2} \backslash Y_{2}$. Then we see that the former is a subdivision of the latter on $X_{2} \backslash\left(Y_{2} \cup \overline{W_{2}}\right)$ and, on the contrary, the latter is a subdivision of the former on $U_{2} \backslash Y$. Hence the restriction of the join $J\left(U_{2}^{*}, U^{*}\right)$ to the open set $X_{2} \backslash Y_{2}$ can be patched with the restriction of $U_{2}^{*}$ to the open set $X_{2} \backslash \overline{W_{2}}$, and they form a subdivision $V_{2}$ of $X_{2}$. Since $U_{1} \backslash Y \subset X^{*}$, we see that $U^{*}$ and hence $V_{2}$ is equal to $U_{1} \backslash Y$ over $U_{2} \backslash Y$. On the other hand, since $X \subset X_{2} \backslash \overline{W_{2}}$ and $I^{\prime}$ is unitary on $X \subset U_{2}, X$ is contained in $V_{2}$. Hence the fan $V_{2}$ over $X_{2}$ is equal to $U_{1}$ 
over $U_{2}=\left(U_{2} \backslash Y\right) \cup X$, and we can patch it with $X_{1}$. The resulting fan $X_{3}$ satisfies the conditions of the theorem.

Now we prove that we can subdivide $X_{1}$ and $X_{2}$ so that the conditions (1) and (2) are satisfied. By Theorem 4.6, there exists a blowup $X_{2}^{\prime}$ of $X_{2}$ which is quasi-dominant over $X_{1}$ and contains $D_{X_{2}, X_{1}}$. Then

$$
U_{2}:=\left\{\sigma \in X_{2}^{\prime} ; \text { there exists } \tau \in X_{1} \text { such that } \sigma \subset \tau\right\}
$$

is an open subset of $X_{2}^{\prime}$ and is equal to $J\left(X_{1}, X_{2}^{\prime}\right)$. Again by Theorem 4.6, there exists a blowup $X_{1}^{\prime}$ of $X_{1}$ quasi-dominant over $X_{2}^{\prime}$ and $D_{X_{1}, X_{2}^{\prime}} \subset X_{1}^{\prime}$. We set

$$
U_{1}:=\left\{\tau \in X_{1}^{\prime} \text {; there exists } \rho \in X_{2}^{\prime} \text { such that } \tau \subset \rho\right\} \text {. }
$$

Since $\mathrm{ZR}\left(U_{1}\right)=\mathrm{ZR}\left(X_{1}\right) \cap \mathrm{ZR}\left(X_{2}^{\prime}\right)=\mathrm{ZR}\left(U_{2}\right), U_{1}$ is a subdivision of $U_{2}$. We replace $X_{1}$ by $X_{1}^{\prime}$ and $X_{2}$ by $X_{2}^{\prime}$. Then the new $X=X_{1} \cap X_{2}$ contains the original $X$. Since $X_{1}^{\prime}$ is a blowup of the original $X_{1}$ along a polyhedral ideal $I$ which is unitary on $X$, we retain the relation $X_{1} \backslash X \subset X^{*}$ if we replace $X^{*}$ by its blowup at the maximal extension of $I \mid\left(X_{1} \cap X^{*}\right)$.

Next, we make them satisfy (3) keeping (1) and (2). Since $W_{1} \subset X_{1} \backslash X \subset X_{1} \cap X^{*}, W_{1}$ and $Y$ are disjoint closed subsets of $U_{1}$. If $V:=\overline{W_{2}} \cap Y_{2}$ is not empty, then this is a closed subset of $X_{2}$ contained in $X_{2} \backslash U_{2}$. By Lemma 3.6, the closures of $W_{1}$ and $Y$ are disjoint in the blowup $X_{2}^{\prime}$ of $X_{2}$ at $V$. (3) is satisfied if we replace $X_{2}$ by $X_{2}^{\prime}$. Since the center of the blowup is outside $U_{2}, X_{1}$ is still quasi-dominant over $X_{2}$.

Finally, we make (4) satisfied. By Theorem 4.6, we can make $X^{*}$ quasi-dominant over $X_{2}$ by a blowup. Here we can take the center of the blowup outside $X_{1} \cap X^{*}$, since $v \in \mathrm{ZR}(M)$ dominates no cones in $X_{2}$ if it dominates a point $\sigma$ in an open subset $X_{1} \cap X^{*}$ of $X^{*}$ and if $\sigma \in X_{1} \backslash U_{1}$. Hence, there is no change in the relation $X_{1} \backslash X \subset X^{*}$. The conditions (1) and (2) are independent of $X^{*}$. (3) is also satisfied, since the new $Y$ is contained in the original $Y$.

Proof of Theorem 4.1. Let $X$ be a finite fan. For any $v \in \mathrm{ZR}(M)$, there exists a fan $X_{v}$ such that $X \subset X_{v}, v \in \mathrm{ZR}\left(X_{v}\right)$ and $X_{v} \backslash X$ is contained in a projective fan by Theorem 4.7. Since $\mathrm{ZR}(M)$ is compact by Theorem 2.5 , there exist finite elements $v_{1}, \ldots, v_{s} \in \mathrm{ZR}(M)$ with

$$
\mathrm{ZR}(M)=\mathrm{ZR}\left(X_{v_{1}}\right) \cup \cdots \cup \mathrm{ZR}\left(X_{v_{s}}\right) .
$$

By applying Theorem 4.8, we construct inductively a sequence of finite fans $X_{1}=$ $X_{v_{1}}, X_{2}, \ldots, X_{s}$ with

$$
\mathrm{ZR}\left(X_{i}\right)=\operatorname{ZR}\left(X_{v_{1}}\right) \cup \cdots \cup \mathrm{ZR}\left(X_{v_{i}}\right)
$$

and $X_{i-1} \cap X_{v_{i}} \subset X_{i}$ for $i=2, \ldots, s$. Then $X^{\prime}:=X_{s}$ is complete by Theorem 4.3 and contains $X$.

5. Compactifications of real fans. In this section, we will prove the compactification theorem for real fans. Indeed, we prove it for $\boldsymbol{k}$-fans for an arbitrary subfield $\boldsymbol{k}$ of $\boldsymbol{R}$. In the case of rational fans, we used the lattice $M$ for blowups. However, we cannot use it in the general case. 
Let $M$ and $N$ be free $Z$-modules of rank $r \geq 0$ as in the previous sections. We fix a subfield $\boldsymbol{k}$ of $\boldsymbol{R}$. Let $N_{\boldsymbol{k}}:=N \otimes \boldsymbol{k}$ and $M_{\boldsymbol{k}}:=M \otimes \boldsymbol{k}$. A cone $\sigma$ in $N_{\boldsymbol{R}}$ is said to be a $\boldsymbol{k}$-cone if it is generated by a finite subset of $N_{\boldsymbol{k}}$. A real fan $X$ is said to be a $\boldsymbol{k}$-fan if every $\sigma \in X$ is a $\boldsymbol{k}$-cone. In particular, $\boldsymbol{R}$-fans are real fans and $\boldsymbol{Q}$-fans are usual fans. Although our theory does not depend on the field $\boldsymbol{k}$, it is an interesting problem to find the properties of $\boldsymbol{k}$-fans which depend on the field $\boldsymbol{k}$.

We set $\boldsymbol{k}_{0}:=\{a \in \boldsymbol{k} ; a \geq 0\}$. Let $C \subset M_{\boldsymbol{R}}$ be the cone generated by a finite subset $\left\{x_{1}, \ldots, x_{s}\right\}$ of $M_{\boldsymbol{k}}$. Then $M_{\boldsymbol{k}} \cap C=\boldsymbol{k}_{0} x_{1}+\cdots+\boldsymbol{k}_{0} x_{s}$ as easily shown by Carathéodory's theorem.

A preorder $\leq$ on $M_{\boldsymbol{k}}$ is said to be $\boldsymbol{k}$-additive if the following conditions are satisfied.

(1) For any $x, y \in M_{k}$, either $x \leq y$ or $y \leq x$ is satisfied.

(2) $x \leq y$ and $y \leq z$ imply $x \leq z$.

(3) If $x \leq y$, then $x+z \leq y+z$ for every $z$.

(4) If $x \leq y$, then $a x \leq a y$ for every $a \in \boldsymbol{k}_{0}$.

We define the Zariski-Riemann space $\mathrm{ZR}\left(M_{\boldsymbol{k}}\right)$ as the set of all $\boldsymbol{k}$-additive preorders of $M_{k}$. We define the weakest topology on $\operatorname{ZR}\left(M_{k}\right)$ such that $\left\{v \in \operatorname{ZR}\left(M_{k}\right) ; 0 \leq_{v} x\right\}$ is open for every $x \in M_{k}$.

We define $L_{k}(v):=\left\{x \in M_{k} ; 0 \leq_{v} x\right\}$ and $L_{k}^{0}(v):=\left\{x \in M_{k} ; 0={ }_{v} x\right\}$ for $v \in \operatorname{ZR}\left(M_{\boldsymbol{k}}\right)$, where we write $x={ }_{v} y$ if $x \leq_{v} y$ and $y \leq_{v} x$. If a cone $C \subset M_{\boldsymbol{R}}$ is generated by $\left\{x_{1}, \ldots, x_{s}\right\} \subset L_{\boldsymbol{k}}^{0}(v)$, then it is easy to see from the above conditions that $M_{\boldsymbol{k}} \cap C$ is contained in $L_{k}^{0}(v)$. We set $\|\sigma\|_{k}:=\left\{v \in \mathrm{ZR}\left(M_{k}\right) ; M_{k} \cap \sigma^{\vee} \subset L_{k}(v)\right\}$ for a convex polyhedral $\boldsymbol{k}$-cone $\sigma$. Then the set of all subsets $\|\sigma\|_{k}$ forms an open basis of the topology of $\mathrm{ZR}\left(M_{\boldsymbol{k}}\right)$. For a $\boldsymbol{k}$-fan $X, \mathrm{ZR}(X)$ is defined as the the union of $\|\sigma\|_{\boldsymbol{k}}$ for $\sigma \in X$. The compactness of $\mathrm{ZR}(X)$ for a finite $\boldsymbol{k}$-fan $X$ is proved similarly to Theorem 2.5.

We denote by $\eta$ the trivial preorder in $\mathrm{ZR}\left(M_{k}\right)$ with $L_{k}(\eta)=M_{k}$.

LEMMA 5.1. Let $v$ be an element of $\mathrm{ZR}\left(M_{k}\right) \backslash\{\eta\}$. Then the closure of $\operatorname{conv}\left(L_{k}(v)\right)$ in $M_{\boldsymbol{R}}$ is equal to that of $L_{k}(v)$, and is a closed half space, i.e., $\left(x_{0} \geq 0\right)$ for an element $x_{0} \in N_{R}$.

PROOF. It suffices to show the convexity of the closure $\overline{L_{k}(v)} \subset M_{R}$ for the first part. Let $x, y$ be elements of $\overline{L_{k}(v)}$. Then there exist sequences $\left\{x_{i}\right\},\left\{y_{i}\right\}$ in $L_{k}(v)$ converging to $x$ and $y$, respectively. We know $t x_{i}+(1-t) y_{i} \in L_{\boldsymbol{k}}(v)$ for $t \in \boldsymbol{k}$ with $0 \leq t \leq 1$ for all $i$. This implies that all the segments $\overline{x_{i} y_{i}}$ are in $\overline{L_{k}(v)}$, and the limit segment $\overline{x y}$ is also in the closed set. Hence $\overline{L_{k}(v)}$ is convex and equal to the closure of $\operatorname{conv}\left(L_{k}(v)\right)$.

We have $M_{k} \cap\left(\operatorname{conv}\left(L_{k}(v)\right)\right)=L_{k}(v)$ by Carathéodory's theorem. Since $v \neq \eta$, we know $\operatorname{conv}\left(L_{k}(v)\right) \neq M_{R}$ by this equality. Since $\overline{L_{k}(v)}$ is the closure of this convex set by the first part, it is not equal to $M_{\boldsymbol{R}}$. Since $L_{k}(v) \cup\left(-L_{k}(v)\right)=M_{k}$, we have $\overline{L_{k}(v)} \cup\left(-\overline{L_{k}(v)}\right)=$ $M_{\boldsymbol{R}}$. Hence the closed convex cone $\overline{L_{k}(v)}$ is a closed half space.

By this lemma, the ranks and the defining sequences are defined for elements in $\mathrm{ZR}\left(M_{\boldsymbol{k}}\right)$ as in Section 2. We say that an element $v \in \mathrm{ZR}\left(M_{\boldsymbol{k}}\right)$ dominates a $\boldsymbol{k}$-cone $\sigma$ if $M_{k} \cap \sigma^{\vee} \subset L_{k}(v)$ and $M_{k} \cap \sigma^{\vee} \cap L_{k}^{0}(v)=M_{k} \cap \sigma^{\perp}$. 
Theorem 4.5 modified for $\boldsymbol{k}$-fans is proved similarly. However, we need a modification for the part in which we used the lattice $M$. It suffices to show the following lemma.

LEMmA 5.2. Assume that $v \in \mathrm{ZR}\left(M_{\boldsymbol{k}}\right)$ dominates $\boldsymbol{k}$-cones $\tau_{1}, \tau_{2}$, and the first generalization $w$ of $v$ dominates a face $\sigma_{1}$ of $\tau_{1}$ and a face $\sigma_{2}$ of $\tau_{2}$. Furthermore, we assume that $\sigma_{1}$ is contained in $\sigma_{2}$. Then there exists a polyhedral ideal I of $F\left(\tau_{1}\right)$ primary at $\tau_{1}$ with the following property. Let $X^{\prime}$ be the blowup of $F\left(\tau_{1}\right)$ along I. Then the cone $\tau_{v} \in X^{\prime}$ dominated by $v$ is contained in $\tau_{2}$.

PROOF. Let $\left\{x_{1}, \ldots, x_{t}\right\} \subset M_{k}$ be a set of generators of $\tau_{2}^{\vee}$. We may assume that $x_{1}, \ldots, x_{l}$ are contained in $\tau_{2}^{\vee} \backslash \tau_{2}^{\perp}$ and $x_{l+1}, \ldots, x_{t}$ are in $\tau_{2}^{\perp}$. Let $s$ be the rank of $v$, and $\left(y_{0}, \ldots, y_{s-1}\right)$ a defining sequence of $v$. Then we have

$$
L_{k}^{0}(v)=\left\{x \in M_{k} ;\left\langle x, y_{i}\right\rangle=0 \text { for } i=0, \ldots, s-1\right\} .
$$

Since $v$ dominates $\tau_{1}, \tau_{2}$, the linear spaces $\tau_{1}^{\perp}$ and $\tau_{2}^{\perp}$ are contained in $L_{\boldsymbol{k}}^{0}(v)_{\boldsymbol{R}}$. Hence $L_{\boldsymbol{k}}^{0}(v)_{\boldsymbol{R}}^{\perp}$ is contained in $N\left(\tau_{1}\right)_{\boldsymbol{R}}:=\tau_{1}+\left(-\tau_{1}\right)$ and $N\left(\tau_{2}\right)_{\boldsymbol{R}}:=\tau_{2}+\left(-\tau_{2}\right)$. We take $x_{0} \in M_{\boldsymbol{k}} \cap$ rel. $\operatorname{int}\left(\tau_{1}^{\vee} \cap \sigma_{1}^{\perp}\right)$ so that $x_{0}+x_{i} \in \tau_{1}^{\vee}$ for every $1 \leq i \leq t$. This is possible by the relation

$$
\tau_{2}^{\vee} \subset \sigma_{2}^{\vee} \subset \sigma_{1}^{\vee}=\tau_{1}^{\vee}+\left(-\operatorname{rel} \operatorname{int}\left(\tau_{1}^{\vee} \cap \sigma_{1}^{\perp}\right)\right) .
$$

If we take $\varepsilon>0$ sufficiently small, then $z=z_{\varepsilon}:=\sum_{j=0}^{s-1} \varepsilon^{j} y_{j}$ is contained in rel. int $\tau_{1} \cap$ rel.int $\tau_{2}$ and $\left\langle x_{i}, z\right\rangle>0$ for all $1 \leq i \leq l$. Now we take elements $a, b$ of $\boldsymbol{k}$ such that $0<a<b,\left\langle x_{0}, z\right\rangle<a$ and $\left\langle x_{0}+x_{i}, z\right\rangle>b$ for $i=1, \ldots, l$. Since $z \in$ rel. int $\tau_{1}$, we see that $\tau_{1}^{\vee} \cap(z \leq b)$ is $\tau_{1}^{\perp}$-bounded, where $(z \leq b)=\left\{x \in M_{\boldsymbol{R}} ;\langle x, z\rangle \leq b\right\}$. We take a point $z_{0}$ in $N_{\boldsymbol{k}} \cap L_{\boldsymbol{k}}^{0}(v)_{\boldsymbol{R}}^{\perp}$ sufficiently near to $z \in L_{\boldsymbol{k}}^{0}(v)_{\boldsymbol{R}}^{\perp}$. Then $z_{0}$ is contained in rel. int $\tau_{1} \cap$ rel. int $\tau_{2}$ and satisfies $\left\langle x_{0}, z_{0}\right\rangle<a$ and

$$
\tau_{1}^{\vee} \cap(z \geq b) \subset \tau_{1}^{\vee} \cap\left(z_{0} \geq(a+b) / 2\right) \subset \tau_{1}^{\vee} \cap(z>a) .
$$

Let $P$ be the convex hull of the union of

$$
\tau_{1}^{\vee} \cap\left(z_{0} \geq(a+b) / 2\right) \text { and } \tau_{1}^{\vee} \cap\left(\left\{x_{0}\right\}+L_{k}^{0}(v)_{R}\right) .
$$

Note that $x_{0}+x_{i}$ is contained in the set on the left hand side for $i=1, \ldots, l$ and in the set on the right hand side for $i=l+1, \ldots, t$. Then $P$ is a $\tau_{1}^{\perp}$-convex subset contained in $\tau_{1}$, and $\tau_{1}^{\vee} \backslash P$ is $\tau_{1}$-bounded, since it is contained in $\tau_{1}^{\vee} \cap(z \leq b)$. Hence $P$ defines a polyhedral ideal $I(P)$ primary at $\tau_{1}$.

The fan $X^{\prime}$ obtained by the blowup of $F\left(\tau_{1}\right)$ along this ideal contains $\rho:=\left(P-x_{0}\right)^{\vee}$ as an element. Then $\tau_{v} \subset \tau_{2}$ since $P \backslash\left\{x_{0}\right\}$ contains $\left\{x_{0}+x_{1}, \ldots, x_{0}+x_{t}\right\}$, and hence $M_{\boldsymbol{k}} \cap \rho^{\vee} \subset L_{\boldsymbol{k}}(v)$. Hence $v$ dominates a face $\tau_{v}$ of $\rho$.

In order to prove the theorem analogous to Theorem 4.7 for $\boldsymbol{k}$-fans, it suffices to show the following lemma.

LEMMA 5.3. Assume that $v \in \mathrm{ZR}\left(M_{\boldsymbol{k}}\right)$ dominates a cone $\tau$, and the first generalization $w$ of $v$ dominates a face $\sigma$ of $\tau$. Then there exists a polyhedral ideal I of $F(\tau)$ primary at $\tau$ with the following properties. For the blowup $X$ of $F(\tau)$ along $I$, the cone $\tau_{v} \in X$ dominated by $v$ satisfies $\tau_{v} \backslash$ rel. int $\tau \subset \sigma$. 
Proof. Let $\left(y_{0}, \ldots, y_{s-1}\right)$ be a defining sequence of $v$. We take $\varepsilon>0$ sufficiently small so that $z=z_{\varepsilon}$ is in rel. int $\tau$. We take an arbitrary $x_{0} \in M_{\boldsymbol{k}} \cap \operatorname{rel}$. int $\left(\tau^{\vee} \cap \sigma^{\perp}\right), a \in \boldsymbol{k}$ greater than $\left\langle x_{0}, z\right\rangle$ and $b \in \boldsymbol{k}$ greater than $a$. As in the proof of Lemma 5.2, we take $z_{0}$ of $N_{\boldsymbol{k}} \cap L_{\boldsymbol{k}}^{0}(v)_{\boldsymbol{R}}^{\perp}$ sufficiently near $z$ so that

$$
\tau^{\vee} \cap(z \geq b) \subset \tau^{\vee} \cap\left(z_{0} \geq(a+b) / 2\right) \subset \tau^{\vee} \cap(z>a) .
$$

We consider the primary polyhedral ideal $I(P)$ defined by the convex closure $P$ of the union of $\tau^{\vee} \cap\left(z_{0} \geq(a+b) / 2\right)$ and $\tau^{\vee} \cap\left(\left\{x_{0}\right\}+\tau^{\perp}\right)$. We will show $\left(P-x_{0}\right)^{\vee} \backslash$ rel. int $\tau \subset \sigma$. Let $u$ be a point of $\left(P-x_{0}\right)^{\vee}$ and $\rho$ the minimal face of $\tau$ which contains $u$. If $u \notin$ rel. int $\tau$, then $\rho \neq \tau$ and $P \cap \rho^{\perp}$ is nonempty. Let $y$ be an element in it. Then since $\langle y, u\rangle=0$, $\left\langle y-x_{0}, u\right\rangle \geq 0$ and $\left\langle x_{0}, u\right\rangle \geq 0$, we have $\left\langle x_{0}, u\right\rangle=0$. Hence $x_{0} \in \rho^{\perp}$ and hence $\rho$ is a face of $\sigma$. Since $M_{k} \cap\left(P-x_{0}\right) \subset L_{k}(v)$ by the construction of $P, v$ dominates a face $\tau_{v}$ of $\left(P-x_{0}\right)^{\vee}$. Then the conditions of the lemma is satisfied for $\tau_{v}$.

Now the following theorem is proved similarly to Theorem 4.1.

THEOREM 5.4. For an arbitrary finite $\boldsymbol{k}$-fan $X$, there exists a complete finite $\boldsymbol{k}$-fan $X^{\prime}$ with $X \subset X^{\prime}$.

If we set $\boldsymbol{k}:=\boldsymbol{R}$, then we get the second proof of Theorem 1.1.

\section{REFERENCES}

[E] G. EWALD, Combinatorial convexity and algebraic geometry, Grand. Texts in Math. 168, Springer-Verlag, New York, 1996.

[F1] K. FujIWARA, Rigid geometry, Lefschetz-Verdier trace formula and Deligne's conjecture, Invent. Math. 127 (1997), 489-533

[F2] W. Fulton, Introduction to toric varieties, Ann. of Math. Stud. 131, Princeton University Press, Princeton, N.J., 1993.

[H] R. Hartshorne, Algebraic Geometry, Grad. Texts in Math. 52, Springer-Verlag, New York-Heidelberg, 1977.

[K] S. Kuroda, The infiniteness of the SAGBI bases for certain invariant rings, Osaka J. Math. 39 (2002), 665680 .

[N1] M. NAGATA, Imbedding of an abstract variety in a complete variety, J. Math. Kyoto Univ. 2 (1962), 1-10.

[N2] M. NAGATA, A generalization of the imbedding problem of an abstract variety in a complete variety, J. Math. Kyoto Univ. 3 (1963), 89-102.

[O1] T. ODA, Convex Bodies and Algebraic Geometry, An Introduction to the Theory of Toric Varieties, Ergebnisse der Math. (3), 15, Springer-Verlag, Berlin, Heidelberg, New York, Tokyo, 1988.

[OP] T. OdA AND H. S. PARK, Linear Gale transforms and Gelfand-Kapranov-Zelevinskij decompositions, Tohoku Math. J. (2) 43 (1991), 375-399.

[S3] B. Sturmfels, Göbner bases and convex polytopes, Univ. Lecture Ser., 8, American Mathematical Society, Provedence, R.I., 1996.

[S1] H. Sumihiro, Equivariant completion, J. Math. Kyoto Univ. 14 (1974), 1-28.

[S2] H. SumihiRo, Equivariant completion II, J. Math. Kyoto Univ. 15 (1975), 573-605.

[Z1] O. ZARISKI, Local uniformization of algebraic varieties, Ann. of Math. (2) 41 (1940), 852-896.

[Z2] O. ZARISKI, The compactness of the Riemann manifold of an abstract field of algebraic functions, Bull. Amer. Math. Soc. 50 (1944), 683-691. 
[ZS] O. Zariski And P. Samuel, Commutative Algebra II, Grad. Texts in Math. 29, Springer-Verlag, New York-Heidelberg, 1975.

FAKULTÄT UND INSTITUT FÜR MATHEMATIK

RUHR-UNIVERSITÄT BOCHUM

UNIVERSITÄTSSTRASSE 150

D-44780 BOCHUM

GERMANY

E-mail address: ewaldfamily@t-online.de
MATHEMATICAL InSTITUTE

TOHOKU UNIVERSITY

SENDAI 980-8578

JAPAN

E-mail address: ishida@math.tohoku.ac.jp 